\title{
CONDITIONS FOR SUPERCONVERGENCE OF HDG METHODS FOR SECOND-ORDER ELLIPTIC PROBLEMS
}

\author{
BERNARDO COCKBURN, WEIFENG QIU, AND KE SHI
}

\begin{abstract}
We provide a projection-based analysis of a large class of finite element methods for second order elliptic problems. It includes the hybridized version of the main mixed and hybridizable discontinuous Galerkin methods. The main feature of this unifying approach is that it reduces the main difficulty of the analysis to the verification of some properties of an auxiliary, locally defined projection and of the local spaces defining the methods. Sufficient conditions for the optimal convergence of the approximate flux and the superconvergence of an element-by-element postprocessing of the scalar variable are obtained. New mixed and hybridizable discontinuous Galerkin methods with these properties are devised which are defined on squares, cubes and prisms.
\end{abstract}

\section{INTRODUCTION}

In this paper, we propose a projection-based a priori error analysis of finite element methods for second-order elliptic problems. The analysis is unifying because it applies to a large class of methods including the hybridized version of most well-known mixed methods as well as several hybridizable discontinuous Galerkin (HDG) methods. The novelty of the approach is that it reduces the whole error analysis to the element-by-element construction of an auxiliary projection satisfying certain orthogonality and approximation properties, and to the verification of very simple inclusion properties of the local spaces defining the methods. For the sake of simplicity, we present our approach in the framework of the following diffusion problem:

$$
\begin{aligned}
\boldsymbol{q}+\nabla u=0 & & \text { in } \Omega, \\
\nabla \cdot \boldsymbol{q}=f & & \text { in } \Omega, \\
u=g & & \text { on } \partial \Omega .
\end{aligned}
$$

Here $\Omega \subset \mathbb{R}^{n}(n=2,3)$ is a bounded polyhedral domain, $f \in L^{2}(\Omega)$ and $g \in$ $H^{\frac{1}{2}}(\partial \Omega)$.

Two ideas led to this approach. The first is that many mixed methods, including the method of Raviart and Thomas (RT), [16, 12, 1, Brezzi, Douglas and Marini, 5, and Brezzi, Douglas, Fortin and Marini, 4, were successfully analyzed by using suitably defined auxiliary projections; see also [6. The second is that both

Received by the editor October 12, 2010 and, in revised form, March 10, 2011.

2010 Mathematics Subject Classification. Primary 35L65, 65M60, 65N30.

Supported in part by the National Science Foundation (Grant DMS-0712955) and by the University of Minnesota Supercomputing Institute. 
mixed and HDG methods can be seen as particular cases of a single, general numerical method uncovered in [10]. This suggested the possibility of using a similar projection-based approach to analyze HDG methods. Recently, this was actually achieved, first for a particular case of HDG methods whose local solvers are defined by the local discontinuous Galerkin method (LDG-H) (defined on simplexes) in 9, and then for the whole family of those methods in [11. In this paper, we continue this effort and show that a single error analysis of many of the methods fitting in the general framework proposed in [10 can be realized.

To better describe our results, let us begin by introducing the general form of the methods we are going to consider; we follow 10. Let $\mathcal{T}_{h}:=\{K\}$ denote a conforming triangulation of $\Omega$, where $K$ is a polyhedral element. We denote the set of faces $F$ of an element $K \in \mathcal{T}_{h}$ by $\mathcal{F}(K)$, and the set of faces $F$ of all elements $K \in \mathcal{T}_{h}$ by $\mathcal{E}_{h}$. The methods we are interested in seek an approximation to $\left(u, \boldsymbol{q},\left.u\right|_{\mathcal{E}_{h}}\right),\left(u_{h}, \boldsymbol{q}_{h}, \widehat{u}_{h}\right)$, in the finite element space $W_{h} \times \boldsymbol{V}_{h} \times M_{h}$, where

$$
\begin{aligned}
V_{h} & :=\left\{\boldsymbol{v} \in \boldsymbol{L}^{2}\left(\mathcal{T}_{h}\right):\left.\boldsymbol{v}\right|_{K} \in \boldsymbol{V}(K), K \in \mathcal{T}_{h}\right\}, \\
W_{h} & :=\left\{w \in L^{2}\left(\mathcal{T}_{h}\right):\left.w\right|_{K} \in W(K), K \in \mathcal{T}_{h}\right\}, \\
M_{h} & :=\left\{\mu \in L^{2}\left(\mathcal{E}_{h}\right):\left.\mu\right|_{F} \in W(F), \quad F \in \mathcal{E}_{h}\right\},
\end{aligned}
$$

and determine it as the only solution of the following weak formulation:

$$
\begin{aligned}
-\left(u_{h}, \nabla \cdot \boldsymbol{v}\right)_{\mathcal{T}_{h}}+\left(\boldsymbol{q}_{h}, \boldsymbol{v}\right)_{\mathcal{T}_{h}}+ & \left\langle\widehat{u}_{h}, \boldsymbol{v} \cdot \boldsymbol{n}\right\rangle_{\partial \mathcal{T}_{h}}=0, \\
+\left(\boldsymbol{q}_{h}, \nabla w\right)_{\mathcal{T}_{h}} & \left\langle\widehat{\boldsymbol{q}}_{h} \cdot \boldsymbol{n}, w\right\rangle_{\partial \mathcal{T}_{h}}=(f, w)_{\mathcal{T}_{h}}, \\
& \left\langle\widehat{\boldsymbol{q}}_{h} \cdot \boldsymbol{n}, \mu\right\rangle_{\partial \mathcal{T}_{h} \backslash \partial \Omega}=0, \\
& \left\langle\widehat{u}_{h}, \mu\right\rangle_{\partial \Omega}=\langle g, \mu\rangle_{\partial \Omega},
\end{aligned}
$$

for all $(w, \boldsymbol{v}, \mu) \in W_{h} \times \boldsymbol{V}_{h} \times M_{h}$. Here we write $(\eta, \zeta)_{\mathcal{T}_{h}}:=\sum_{K \in \mathcal{T}_{h}}(\eta, \zeta)_{K}$, where $(\eta, \zeta)_{D}$ denotes the integral of $\eta \zeta$ over the domain $D \subset \mathbb{R}^{n}$. We also write $(\eta, \zeta)_{\mathcal{T}_{h}}:=\sum_{K \in \mathcal{T}_{h}}\langle\eta, \zeta\rangle_{\partial K}$, where $\langle\eta, \zeta\rangle_{D}$ denotes the integral of $\eta \zeta$ over the domain $D \subset \mathbb{R}^{n-1}$ and $\partial \mathcal{T}_{h}:=\left\{\partial K: K \subset \mathcal{T}_{h}\right\}$. The definition of the method is completed with the definition of the normal component of the numerical trace:

$$
\widehat{\boldsymbol{q}}_{h} \cdot \boldsymbol{n}=\boldsymbol{q}_{h} \cdot \boldsymbol{n}+\alpha\left(u_{h}-\widehat{u}_{h}\right) \quad \text { on } \quad \partial \mathcal{T}_{h} .
$$

By taking particular choices of the local spaces $\boldsymbol{V}(K), W(K)$ and $M(F)$, and the linear local stabilization operator $\alpha$, the different mixed $(\alpha=0)$ and HDG $(\alpha \neq 0)$ methods are obtained.

Our main result is to show that if we can construct, in an element-by-element fashion, an auxiliary projection $\Pi_{u}(\boldsymbol{q}, u):=\left(\boldsymbol{\Pi}_{V} \boldsymbol{q}, \Pi_{W} u\right)$ satisfying certain orthogonality and approximation conditions, and the local spaces $\boldsymbol{V}(K), W(K)$ and $M(F)$, for all the faces $F$ of the element $K$, satisfy some inclusion properties, then the method is well defined and we have the estimates

$$
\begin{aligned}
\left\|\boldsymbol{q}-\boldsymbol{q}_{h}\right\|_{\mathcal{T}_{h}} & \leq 2\left\|\boldsymbol{q}-\boldsymbol{\Pi}_{V} \boldsymbol{q}\right\|_{\mathcal{T}_{h}}, \\
\left\|\Pi_{W} u-u_{h}\right\|_{\mathcal{T}_{h}} & \leq C h\left\|\boldsymbol{q}-\boldsymbol{\Pi}_{V} \boldsymbol{q}\right\|_{\mathcal{T}_{h}},
\end{aligned}
$$

where $\|\cdot\|_{\mathcal{T}_{h}}$ denotes the $L^{2}\left(\mathcal{T}_{h}\right)$-norm.

Note that if the error $\Pi_{W} u-u_{h}$ converges to zero faster than the error $u-u_{h}$, this superconvergence property can be advantageously exploited; see [1, 5, 17, 13, 18. 
Indeed, following [17, 13, 18, we define a new approximation to $u, u_{h}^{*}$, in the space

$$
W_{h}^{*}:=\left\{w \in L^{2}\left(\mathcal{T}_{h}\right):\left.w\right|_{K} \in W^{*}(K), K \in \mathcal{T}_{h}\right\},
$$

as follows. On each element $K \in \mathcal{T}_{h}$, the function $u_{h}^{*}$ is the element of $W^{*}(K)$ such that

$$
\begin{aligned}
\left(\nabla u_{h}^{*}, \nabla \omega\right)_{K} & =-\left(\boldsymbol{q}_{h}, \nabla \omega\right)_{K} \quad \forall \omega \in W^{*}(K):(\omega, 1)_{K}=0, \\
\left(u_{h}^{*}, 1\right)_{K} & =\left(u_{h}, 1\right)_{K} .
\end{aligned}
$$

It is not difficult to prove that $u_{h}^{*}$ is well defined and that we have

$$
\left\|u-u_{h}^{*}\right\|_{\mathcal{T}_{h}} \leq\left\|\Pi_{W} u-u_{h}\right\|_{\mathcal{T}_{h}}+C h\left(\left\|\boldsymbol{q}-\boldsymbol{q}_{h}\right\|_{\mathcal{T}_{h}}+\inf _{\omega \in W_{h}^{*}}\|\nabla(u-\omega)\|_{\mathcal{T}_{h}}\right),
$$

which means that it is possible to define $u_{h}^{*}$ converging to $u$ as fast as $\Pi_{W} u-u_{h}$ converges to zero.

Moreover, we do provide a single template for the choice of the local spaces $\boldsymbol{V}(K), W(K)$ and $M(F)$, and for the stabilization operator $\alpha$ which guarantees the existence of the auxiliary projection $\Pi_{h}$ with the above-mentioned properties. Using this template, we give many examples of superconvergent methods, old and new, fitting our general framework. The old ones are the (hybridized versions of) the main mixed methods, the LDG-H and BMMPR-H methods for simplexes; see [10]. The last method, which had never been analyzed before, is proven to superconverge even though it uses a local stabilization operator $\alpha$ which is different from those of the previous examples.

The new methods are several LDG-H methods for squares, cubes and prisms. The definition of these methods had remained elusive in the last few years and it is thanks to the our projection-based approach that it became clear. It is important to emphasize that, although it is very easy to devise HDG methods that are well defined, it is far from obvious to devise them so that they display the abovementioned superconvergence property. The technique we propose here is a new and effective tool to achieve this goal.

Yet another new method is what seems to be the smallest superconvergent mixed method, on squares and cubes, whose local space $\boldsymbol{V}(K) \times W(K)$ contains the tensorproduct space $\boldsymbol{Q}^{k}(K) \times Q^{k}(K)$. Here, we prove that, by only adding a fixed number (3 in two-space dimensions and 7 in three-space dimensions) of extra basis functions to $\boldsymbol{Q}^{k}(K)$, we obtain the orders of convergence of $k+1$ for the approximate flux $\boldsymbol{q}_{h}$ and of $k+2$ for postprocessing $u_{h}^{*}$, provided $k \geq 1$. This new method has convergence properties similar to those of the corresponding RT method (see [6]), but uses significantly smaller spaces.

The rest of the paper is organized as follows. In Section 2, we describe the conditions on the auxiliary projection $\Pi_{h}$ and the local spaces associated with our finite element methods and present our a priori error estimates. In Section 3, we describe a template for the devising of superconvergent HDG methods. In Section 4 , we use it to we give various particular examples of hybridized mixed and HDG methods with superconvergent properties. In Section 5, we provide a detailed proof of the a priori error estimates. We then end with some extensions and concluding remarks in Section 6. 


\section{MAin RESUlts}

In this section we show how an a priori error analysis of the HDG methods can be reduced to the verification of a few conditions on the local spaces and on some properties of an associated, auxiliary projection $\Pi_{h}$ defined in an element-byelement fashion.

2.1. A priori error estimates. The main idea of our error analysis is to estimate the projection of the errors $\Pi_{h}\left(\boldsymbol{q}-\boldsymbol{q}_{h}, u-u_{h}\right)$ and then deduce bounds of the $L^{2}(\Omega)$-norm of the errors $\boldsymbol{q}-\boldsymbol{q}_{h}, u-u_{h}$ and $u-u_{h}^{*}$.

2.1.1. Estimate of $\boldsymbol{q}-\boldsymbol{q}_{h}$. Our first result gives an estimate of the projection of the error $\boldsymbol{\Pi}_{V} \boldsymbol{q}-\boldsymbol{q}_{h}$ solely in terms of the approximation error of the projection $\boldsymbol{q}-\boldsymbol{\Pi}_{V} \boldsymbol{q}$. To state it, we need to describe our assumptions on the projection $\Pi_{h}$ and on the local finite element spaces $\boldsymbol{V}(K), W(K)$, and $M(F)$.

\section{Assumptions A:}

- Orthogonality properties of $\Pi_{h}$. On each element $K$, there exist a projection $\Pi_{h}(\boldsymbol{q}, u)=\left(\boldsymbol{\Pi}_{V} \boldsymbol{q}, \Pi_{W} u\right) \in \boldsymbol{V}(K) \times W(K)$ satisfying the following properties:

$(A .1)\left(\boldsymbol{\Pi}_{V} \boldsymbol{q}, \boldsymbol{v}\right)_{K}=(\boldsymbol{q}, \boldsymbol{v})_{K}$ for all $\boldsymbol{v} \in \nabla W(K)$.

(A.2) $\left(\Pi_{W} u, w\right)_{K}=(u, w)_{K}$ for all $w \in \nabla \cdot V(K)$.

(A.3) For all faces $F$ of the element $K$,

$\left\langle\Pi_{V} \boldsymbol{q} \cdot \boldsymbol{n}+\alpha\left(\Pi_{W} u\right), \mu\right\rangle_{F}=\left\langle\boldsymbol{q} \cdot \boldsymbol{n}+\alpha\left(P_{M} u\right), \mu\right\rangle_{F} \quad$ for all $\mu \in M(F)$.

We also need to assume suitable relations between the traces on the faces $F$ of the local spaces $\boldsymbol{V}(K)$ and $W(K)$ with the local space $M(F)$.

- Properties of the traces of the local spaces. For each element $K$, and for any of its faces $F$ :

(A.4) $\left.\boldsymbol{V}(K) \cdot \boldsymbol{n}\right|_{F} \subset M(F)$.

$\left.(A .5) W(K)\right|_{F} \subset M(F)$.

Here, $\left.\boldsymbol{V}(K) \cdot \boldsymbol{n}\right|_{F}$ denotes the space of the traces of normal components of functions of $\boldsymbol{V}(K)$ on the face $F$ of $K$. Similarly, $\left.W(K)\right|_{F}$ denotes the space of traces of functions of $W(K)$ on the face $F$.

Finally, we need a simple assumption reflecting the stabilizing role of the linear operator $\alpha$.

- The semi-positivity property of $\alpha$. For each element $K$ and any of its faces $F$, (A.6) $\langle\alpha(\mu), \mu\rangle_{F} \geq 0$ for all $\mu \in M(F)$.

We are now ready to state our first result. In what follows, we use $\|\cdot\|_{k, D},|\cdot|_{k, D}$ to denote the standard norm and seminorm on any Sobolev space $H^{k}(D)$, respectively. For simplicity, we use $\|\cdot\|_{D}$ to denote the $L^{2}(D)$-norm on any $D$.

Theorem 2.1. Suppose that the Assumptions A are satisfied. Then we have

$$
\left\|\boldsymbol{\Pi}_{V} \boldsymbol{q}-\boldsymbol{q}_{h}\right\|_{\mathcal{T}_{h}} \leq\left\|\boldsymbol{q}-\boldsymbol{\Pi}_{V} \boldsymbol{q}\right\|_{\mathcal{T}_{h}} .
$$

Note that, since this implies that

$$
\left\|\boldsymbol{q}-\boldsymbol{q}_{h}\right\|_{\mathcal{T}_{h}} \leq 2\left\|\boldsymbol{q}-\boldsymbol{\Pi}_{V} \boldsymbol{q}\right\|_{\mathcal{T}_{h}}
$$

the quality of the approximation $\boldsymbol{q}_{h}$ depends on the approximation properties of the first component of the projection $\Pi_{h}$ only. 
2.1.2. Estimate of $u-u_{h}$. Our next result shows that $\Pi_{W} u-u_{h}$ can also be controlled solely in terms of the approximation error of the projection $\boldsymbol{q}-\boldsymbol{\Pi}_{V} \boldsymbol{q}$.

It is valid under a typical elliptic regularity property we state next. We assume that, for any given $\eta \in L^{2}(\Omega)$, we have

$$
\|\phi\|_{2, \Omega}+\|\boldsymbol{\theta}\|_{1, \Omega} \leq C\|\eta\|_{\Omega},
$$

where $C$ only depends on the domain $\Omega$, and $(\boldsymbol{\theta}, \phi)$ is the solution of the dual problem:

$$
\begin{aligned}
& \boldsymbol{\theta}+\nabla \phi=0 \text { in } \Omega, \\
& \nabla \cdot \boldsymbol{\theta}=\eta \quad \text { in } \Omega \text {, } \\
& \phi=0 \quad \text { on } \partial \Omega \text {. }
\end{aligned}
$$

We also need a couple of additional assumptions.

\section{Assumptions B:}

The first is an approximation property of a projection $\Pi_{h}^{*}(\boldsymbol{q}, u)=\left(\boldsymbol{\Pi}_{V}^{*} \boldsymbol{q}, \Pi_{W}^{*} u\right)$ which satisfies the assumptions $(A .1),(A .2)$, and $(A .3)$ where the local stabilization operator $\alpha(\cdot)$ is replaced by its dual $\alpha^{*}(\cdot)$, that is, by the linear function defined by

$$
\left\langle\eta, \alpha^{*}(\mu)\right\rangle_{F}=\langle\alpha(\eta), \mu\rangle_{F} \quad \text { for all } \quad \eta, \mu \in M(F) \forall F \in \mathcal{F}(K) .
$$

- The approximation property of the projection $\Pi_{h}^{*}$. For each element $K$ and any $(\boldsymbol{q}, u) \in \boldsymbol{H}^{1}(K) \times H^{2}(K)$,

(B.1) $\left\|\boldsymbol{\Pi}_{V}^{*} \boldsymbol{q}-\boldsymbol{q}\right\|_{K} \leq C_{a p p}^{*} h_{K}\left(|u|_{1, K}+|\boldsymbol{q}|_{1, K}\right)$.

The second assumption is a condition on the local space $W(K)$.

- The local space $W(K)$ is not too small. For each element $K$, we have that (B.2) $\boldsymbol{P}^{0}(K) \subset \nabla W(K)$.

Here $\boldsymbol{P}^{0}(K):=\left[P^{0}(K)\right]^{n}$ and $P^{0}(K)$ is the space of constants defined on $K$.

We are now ready to state our second result.

Theorem 2.2. Suppose that the Assumptions $A$ and $B$ are satisfied. Also, suppose that the elliptic regularity property (2.1) holds. Then we have

$$
\left\|\Pi_{W} u-u_{h}\right\|_{\mathcal{T}_{h}} \leq C h\left\|\boldsymbol{q}-\boldsymbol{\Pi}_{V} \boldsymbol{q}\right\|_{\mathcal{T}_{h}},
$$

for some constant $C$ depending on $C_{a p p}^{*}$ but independent of $h$ and the exact solution.

From this result, we immediately get that

$$
\left\|u-u_{h}\right\|_{\mathcal{T}_{h}} \leq\left\|u-\Pi_{W} u\right\|_{\mathcal{T}_{h}}+C h\left\|\boldsymbol{q}-\Pi_{V} \boldsymbol{q}\right\|_{\mathcal{T}_{h}},
$$

and we see that the quality of the approximation $u_{h}$ only depends on the approximation error of the projection.

2.1.3. Estimate of $u-u_{h}^{*}$. Note that if the second term of the above right-hand side converges faster than the first, the convergence of $u_{h}$ to $\Pi_{W} u$ is faster than that of $u_{h}$ to $u$. As mentioned before, we can take advantage of this superconverge result to show that the postprocessing $u_{h}^{*}$ defined by (1.4) converges to $u$ as fast as $u_{h}$ superconverges to $\Pi_{W} u$. For that purpose, we need the following assumption. 
Assumption $C$ :

- The local space $\boldsymbol{V}(K)$ is not too small. For each element $K$, (C.1) $P^{0}(K) \subset \nabla \cdot \boldsymbol{V}(K)$.

We can now state our third and last result.

Theorem 2.3. Suppose that the Assumptions A, B, and $C$ are satisfied. Then, we have

$$
\left\|u-u_{h}^{*}\right\|_{\mathcal{T}_{h}} \leq\left\|\Pi_{W} u-u_{h}\right\|_{\mathcal{T}_{h}}+C h\left(\left\|\boldsymbol{q}-\boldsymbol{\Pi}_{V} \boldsymbol{q}\right\|_{\mathcal{T}_{h}}+\inf _{\omega \in W_{h}^{*}}\|\nabla(u-\omega)\|_{\mathcal{T}_{h}}\right) .
$$

\section{A template For the CONStruction of SUPERConVERGent Methods}

In this section, we provide a particular way of constructing HDG methods satisfying Assumptions $A, B$ and $C$. It turns out that all of the already known methods in the literature (which we also gather in this section) can be constructed by using this template. Moreover, all of the new superconvergent methods we introduce here were obtained by using it.

3.1. The choice of the local spaces and the stabilization operator. To construct our superconvergent methods, we pick an arbitrary element $K \in \mathcal{T}_{h}$, and proceed as follows:

Step 1: The local space $\boldsymbol{V}(K) \times W(K)$. We begin by taking a local space $\boldsymbol{V}(K) \times$ $W(K)$ such that

$$
\begin{aligned}
& \boldsymbol{P}^{0}(K) \subset \nabla W(K) \subset \boldsymbol{V}(K), \\
& P^{0}(K) \subset \nabla \cdot \boldsymbol{V}(K) \subset W(K) .
\end{aligned}
$$

Step 2: The local space $M(F)$. Then, for each face $F$ of the element $K$, we find a space $M(F)$ such that

$$
\begin{array}{r}
\left.\boldsymbol{V}(K) \cdot \boldsymbol{n}\right|_{F} \subset M(F), \\
\left.W(K)\right|_{F} \subset M(F) .
\end{array}
$$

This choice has to be made so that

$$
\sum_{F \in \mathcal{F}(K)} \operatorname{dim} M(F) \leq(\operatorname{dim} \boldsymbol{V}(K)-\operatorname{dim} \nabla W(K))+(\operatorname{dim} W(K)-\nabla \cdot \boldsymbol{V}(K)) .
$$

Step 3: The auxiliary local space $\widetilde{\boldsymbol{V}}(K) \times \widetilde{W}(K)$. Next, we find an auxiliary space $\widetilde{\boldsymbol{V}}(K) \times \widetilde{W}(K)$ satisfying

$$
\begin{aligned}
\nabla W(K) & \subset \widetilde{\boldsymbol{V}}(K) \subset \boldsymbol{V}(K), \\
\nabla \cdot \boldsymbol{V}(K) & \subset \widetilde{W}(K) \subset W(K),
\end{aligned}
$$

such that, if we set

$$
\begin{aligned}
\boldsymbol{V}^{\perp}(K) & :=\left\{\boldsymbol{v} \in \boldsymbol{V}(K):(\boldsymbol{v}, \widetilde{\boldsymbol{v}})_{K}=0\right. & \forall \widetilde{\boldsymbol{v}} \in \widetilde{\boldsymbol{V}}(K)\}, \\
W^{\perp}(K) & :=\left\{w \in W(K):(w, \widetilde{w})_{K}=0\right. & \forall \widetilde{w} \in \widetilde{W}(K)\},
\end{aligned}
$$

we have that

$$
\sum_{F \in \mathcal{F}(K)} \operatorname{dim} M(F)=\operatorname{dim} \boldsymbol{V}^{\perp}(K)+\operatorname{dim} W^{\perp}(K),
$$


and that

$$
\begin{array}{rlrl}
\left\|\boldsymbol{v}^{\perp}\right\|_{K} & \leq C_{V} h_{K}^{1 / 2}\left\|\boldsymbol{v}^{\perp} \cdot \boldsymbol{n}\right\|_{\partial K_{V}} & & \text { for all } \boldsymbol{v}^{\perp} \in \boldsymbol{V}^{\perp}(K), \\
\left\|w^{\perp}\right\|_{K} \leq C_{W} h_{K}^{1 / 2}\left\|w^{\perp}\right\|_{\partial K_{W}} & \text { for all } w^{\perp} \in W^{\perp}(K),
\end{array}
$$

for some subsets $\partial K_{V}$ and $\partial K_{W}$ of $\mathcal{F}(K)$.

Step 4: The stabilization operator $\alpha$. Finally, we pick the local stabilization operator $\alpha$ such that

$$
\begin{aligned}
& \langle\alpha(\eta), \mu\rangle_{F}=\langle\eta, \alpha(\mu)\rangle_{F} \quad \text { for all } \eta, \mu \in M(F), \\
& \left\langle\alpha\left(w^{\perp}\right), w^{\perp}\right\rangle_{\partial K} \geq C_{\alpha}\left\|w^{\perp}\right\|_{\partial K_{W}}^{2} \text { for all } w^{\perp} \in W^{\perp}(K) .
\end{aligned}
$$

Let us briefly discuss the most difficult points of the above steps. The first concerns the inequality (3.3) which states, roughly speaking, that the kernel of the divergence operator in $\boldsymbol{V}(K)$ has to be big enough. Indeed, if we assume that $W(K) \supset P^{0}(K)$, such an inequality can be rewritten as

$$
\sum_{F \in \mathcal{F}(K)} \operatorname{dim} M(F) \leq \operatorname{dim}\{\boldsymbol{v} \in \boldsymbol{V}(K): \nabla \cdot \boldsymbol{v}=0\}+1 .
$$

As a consequence, if this condition is not satisfied, we can simply add to the basis of the space $\boldsymbol{V}(K)$ divergence-free functions whose normal component on each the faces $F$ of $K$ lies on $M(F)$. Note that the more faces an element $K$ has, the harder is to satisfy this inequality, and so, the more of such basis functions will have to be added.

The second concerns the construction of the auxiliary space $\widetilde{\boldsymbol{V}}(K) \times \widetilde{W}(K)$. Once the inequality (3.3) is satisfied, the existence of at least one auxiliary space satisfying the inclusion properties (3.4) and the equality (3.6) is guaranteed, assuming that the inclusion properties in Step 1 and the inequality in Step 2 are satisfied. However, the inequalities (3.7) still need to be satisfied. This means that the above-mentioned additional functions have to be controlled by their normal components.

3.2. Verification of the Assumptions $A, B$, and $C$. We claim that the HDG method determined by the above local spaces and stabilization operator does satisfy Assumptions $A, B$, and $C$. Let us show that this is indeed the case.

It is easy to see that Assumption (A.4) is nothing but condition (3.2a), that Assumption (A.5) is nothing but condition (3.2b), that Assumption (A.6) follows from condition (3.8b), that Assumption (B.2) is nothing but the first inclusion in condition (3.1a), and that Assumption (C.1) is nothing but the first inclusion in condition (3.1b).

To verify the remaining Assumptions, we must introduce an auxiliary projection $\Pi_{h}$. We define, for any element of $\boldsymbol{H}^{1}(K) \times H^{1}(K),(\boldsymbol{q}, u)$, the projection $\Pi_{h}(\boldsymbol{q}, u):=\left(\boldsymbol{\Pi}_{V} \boldsymbol{q}, \Pi_{W} u\right)$ as the element of $\boldsymbol{V}(K) \times W(K)$ satisfying the equations

$$
\begin{aligned}
\left(\boldsymbol{\Pi}_{V} \boldsymbol{q}, \widetilde{\boldsymbol{v}}\right)_{K} & =(\boldsymbol{q}, \widetilde{\boldsymbol{v}})_{K} & & \forall \widetilde{\boldsymbol{v}} \in \widetilde{\boldsymbol{V}}(K), \\
\left(\Pi_{W} u, \widetilde{w}\right)_{K} & =(u, \widetilde{w})_{K} & & \forall \widetilde{w} \in \widetilde{W}(K), \\
\left\langle\boldsymbol{\Pi}_{V} \boldsymbol{q} \cdot \boldsymbol{n}+\alpha\left(\Pi_{W} u\right), \mu\right\rangle_{F} & =\left\langle\boldsymbol{q} \cdot \boldsymbol{n}+\alpha\left(P_{M} u\right), \mu\right\rangle_{F} & & \forall \mu \in M(F),
\end{aligned}
$$

for all faces $F$ of the element $K$.

If this projection were well defined, Assumption (A.1) would follow from the first equation defining the projection, 3.9a), and from the first inclusion in condition 
(3.4a); Assumption (A.2) would follow from the second equation defining the projection, (3.9b), and from the first inclusion in condition (3.4b); and Assumption (A.3) from the third equation defining the projection, (3.9c). Thus, it only remains to prove that the projection is well defined and that it satisfies Assumption (B.1).

Note that since we are assuming that the stabilization operator $\alpha$ is self-adjoint, see condition (3.8a), we have that $\Pi_{h}^{*}=\Pi_{h}$. Note also that, by condition (3.6), the system of equations defining the projection $\Pi_{h}$ is square. Hence, it is well defined if and only if, when $(\boldsymbol{q}, u)=(\mathbf{0}, 0)$, we have that $\Pi_{h}(\boldsymbol{q}, u)=(\mathbf{0}, 0)$. As a consequence, both the existence of the projection $\Pi_{h}$ as well as Assumption (B.1) follow from the approximation result we state next.

To do it, we need to introduce some notation. We denote by $\left(\boldsymbol{P}_{V}, P_{W}, P_{\widetilde{W}}\right)$ the $L^{2}$-projection into the local space $\boldsymbol{V}(K) \times W(K) \times \widetilde{W}(K)$. For any face $F$ of the element $K$, we set

$$
\|\alpha\|_{F}:=\sup _{\mu \in M(F) \backslash\{0\}}\|\alpha(\mu)\|_{F} /\|\mu\|_{F}
$$

and define $\|\alpha\|_{D}:=\max _{F \in D}\|\alpha\|_{F}$ where $D$ is any union of faces of $K$. Finally, we set, for $W^{\perp}(K) \neq\{0\}, R_{W^{\perp}}:=\sup _{w \in W^{\perp}(K) \backslash\{0\}} h_{K}^{1 / 2}\|w\|_{\partial K} /\|w\|_{K}$.

We are now ready to state our result.

Theorem 3.1. We have

$$
\begin{aligned}
\left\|\boldsymbol{q}-\boldsymbol{\Pi}_{V} \boldsymbol{q}\right\|_{K} \leq & \left\|\boldsymbol{q}-\boldsymbol{P}_{V} \boldsymbol{q}\right\|_{K}+\mathrm{C}_{1} h_{K}^{1 / 2}\left\|\left(\boldsymbol{q}-\boldsymbol{P}_{V} \boldsymbol{q}\right) \cdot \boldsymbol{n}\right\|_{\partial K_{V}} \\
& +\mathrm{C}_{2} h_{K}\left\|\nabla \cdot \boldsymbol{q}-\boldsymbol{P}_{\widetilde{W}} \nabla \cdot \boldsymbol{q}\right\|_{K}+\mathrm{C}_{3} h_{K}^{1 / 2}\left\|u-P_{W} u\right\|_{\partial K_{W}}, \\
\left\|u-\Pi_{W} u\right\|_{K} \leq & \left\|u-P_{W} u\right\|_{K}+\mathrm{C}_{4} h_{K}^{1 / 2}\left\|u-P_{W} u\right\|_{\partial K} \\
& +\mathrm{C}_{5} h_{K}\left\|\nabla \cdot \boldsymbol{q}-\boldsymbol{P}_{\widetilde{W}} \nabla \cdot \boldsymbol{q}\right\|_{K}
\end{aligned}
$$

where $\mathrm{C}_{1}:=C_{V}, \mathrm{C}_{2}:=0, \mathrm{C}_{3}:=\mathrm{C}_{V}\|\alpha\|_{\partial K_{V}}, \mathrm{C}_{4}:=0$, and $\mathrm{C}_{5}:=0$ whenever $\widetilde{W}(K)=$ $W(K)$. Otherwise,

$$
\begin{aligned}
& \mathrm{C}_{1}:=C_{V}, \quad \mathrm{C}_{2}:=C_{V} C_{W} R_{W^{\perp}}\|\alpha\|_{\partial K_{V}} / C_{\alpha}, \quad \mathrm{C}_{5}:=C_{W}^{2} / C_{\alpha}, \\
& \mathrm{C}_{4}:=\mathrm{C}_{5} R_{W^{\perp}}\|\alpha\|_{\partial K}, \quad \mathrm{C}_{3}:=\mathrm{C}_{V}\|\alpha\|_{\partial K_{V}}\left(1+R_{W^{\perp}}^{2} C_{W}\|\alpha\|_{\partial K} / C_{\alpha}\right) .
\end{aligned}
$$

This result contains the information of how the choice of local spaces and stabilization operator affects the approximation properties of the projection. It indicates how to choose them to obtain optimal orders of convergence. Let us focus our discussion on the estimate of $\left\|\boldsymbol{q}-\boldsymbol{\Pi}_{V} \boldsymbol{q}\right\|_{K}$ as it is the only relevant one for the convergence properties described in the theorems of Section 2.

Note that if $\widetilde{W}(K)$ coincides with $W(K)$, then $\Pi_{W}$ is the $L^{2}$-projection onto $W(K)$ and we can take $\alpha=0$ on all faces. So we have $C_{2}=C_{3}=0$. When $\widetilde{W}(K)$ is a strict subdomain of $W(K)$, this is still true if we have that $\partial K_{V} \cap \partial K_{W}=\emptyset$ and if we take $\alpha$ in such a way that $\|\alpha\|_{\partial K_{V}}=0$. In these two cases, the approximation properties of $\boldsymbol{\Pi}_{V}$ are, roughly speaking, those of the $L^{2}$-projection $\boldsymbol{P}_{V}$.

In the general case, it is enough to take the stabilization operator $\alpha$ such that $\|\alpha\|_{\partial K_{V}}$ and $\|\alpha\|_{\partial K} / C_{\alpha}$ are uniformly bounded to ensure that the constants $\mathrm{C}_{1}, \mathrm{C}_{2}$ and $\boldsymbol{C}_{3}$ are independent of $\alpha$. In this case, we see that $\boldsymbol{I} \boldsymbol{d}-\boldsymbol{\Pi}_{V}$ converges to zero, roughly speaking, as fast as the projections $\boldsymbol{I} \boldsymbol{d}-\boldsymbol{P}_{V}, h_{K}\left(I d-P_{\widetilde{W}}\right)$ and $I d-P_{W}$ do. In particular, by the inclusion conditions (3.1), we readily get that

$$
\left\|\boldsymbol{q}-\boldsymbol{\Pi}_{V} \boldsymbol{q}\right\|_{K} \leq\left(1+C \mathrm{C}_{1}+2 \mathrm{C}_{2}\right) h_{K}|\boldsymbol{q}|_{1, K}+C \mathrm{C}_{3} h_{K}|u|_{1, K},
$$


and Assumption (B.1) is verified, as claimed. Here, the constant $C$ depends on the shape regularity of the element $K$ and the dimension of the local space $\boldsymbol{V}(K) \times$ $W(K)$.

3.3. Proof of the approximation properties of $\Pi_{h}$, Theorem 3.1. To prove the estimates of Theorem 3.1. we follow 11. The idea is to estimate the quantities $\delta_{\boldsymbol{q}}:=\Pi_{V} \boldsymbol{q}-\boldsymbol{P}_{V} \boldsymbol{q}$ and $\delta_{u}:=\Pi_{W} u-P_{W} u$, and then use the triangle inequality to obtain the desired estimates. We proceed in three steps.

Step 1: The equations for $\delta_{q}$ and $\delta_{u}$. By the equations defining the projection $\Pi_{h}$, (3.9), we have that

$$
\begin{aligned}
\left(\boldsymbol{\delta}_{\boldsymbol{q}}, \widetilde{\boldsymbol{v}}\right)_{K} & =0 & & \forall \widetilde{\boldsymbol{v}} \in \widetilde{\boldsymbol{V}}(K), \\
\left(\delta_{u}, \widetilde{w}\right)_{K} & =0 & & \forall \widetilde{w} \in \widetilde{W}(K), \\
\left\langle\boldsymbol{\delta}_{\boldsymbol{q}} \cdot \boldsymbol{n}+\alpha\left(\delta_{u}\right), \mu\right\rangle_{F} & =\left\langle\boldsymbol{I}_{\boldsymbol{q}} \cdot \boldsymbol{n}+\alpha\left(I_{u}\right), \mu\right\rangle_{F} & & \forall \mu \in M(F),
\end{aligned}
$$

for all faces $F$ of the element $K$. Here $\boldsymbol{I}_{\boldsymbol{q}}:=\boldsymbol{q}-\boldsymbol{P}_{V} \boldsymbol{q}$ and $I_{u}:=P_{M} u-P_{W} u$.

Step 2: The estimate of $\delta_{u}$. Next, we obtain an estimate of $\delta_{u}$. By the definition of $W^{\perp}(K)$, (3.5b), we see that $\delta_{u} \in W^{\perp}(K)$, by the equation (3.10b). If $W^{\perp}(K)=$ $\{0\}$, then

$$
\left\|\delta_{u}\right\|_{K}=0
$$

If $W^{\perp}(K) \neq\{0\}$, we claim that $\delta_{u}$ is the element of $W^{\perp}(K)$ satisfying

$$
\left\langle\alpha\left(\delta_{u}\right), w\right\rangle_{\partial K}=\left(\left(\boldsymbol{I} \boldsymbol{d}-\boldsymbol{P}_{\widetilde{W}}\right) \nabla \cdot \boldsymbol{q}, w\right)_{K}+\left\langle\alpha\left(I_{u}\right), w\right\rangle_{\partial K} \quad \forall w \in W^{\perp}(K) .
$$

Taking $w:=\delta_{u}$ and applying the Cauchy-Schwarz inequality, we get

$$
\begin{aligned}
\left\langle\alpha\left(\delta_{u}\right), \delta_{u}\right\rangle_{\partial K} & \leq\left\|\left(\boldsymbol{I} \boldsymbol{d}-\boldsymbol{P}_{\widetilde{W}}\right) \nabla \cdot \boldsymbol{q}\right\|_{K}\left\|\delta_{u}\right\|_{K}+\left\|\alpha\left(I_{u}\right)\right\|_{\partial K}\left\|\delta_{u}\right\|_{\partial K} \\
& \leq C_{W}\left(h_{K}^{1 / 2}\left\|\left(\boldsymbol{I} \boldsymbol{d}-\boldsymbol{P}_{\widetilde{W}}\right) \nabla \cdot \boldsymbol{q}\right\|_{K}+R_{W^{\perp}}\|\alpha\|_{\partial K}\left\|I_{u}\right\|_{\partial K}\right)\left\|\delta_{u}\right\|_{\partial K_{W}},
\end{aligned}
$$

by the condition (3.7b), and, by the condition (3.8b) on the stabilization operator $\alpha$,

$$
\left\|\delta_{u}\right\|_{\partial K_{W}} \leq \frac{C_{W}}{C_{\alpha}}\left(h_{K}^{1 / 2}\left\|\left(\boldsymbol{I} \boldsymbol{d}-\boldsymbol{P}_{\widetilde{W}}\right) \nabla \cdot \boldsymbol{q}\right\|_{K}+R_{W^{\perp}}\|\alpha\|_{\partial K}\left\|I_{u}\right\|_{\partial K}\right) .
$$

Finally, using once again condition (3.7b), we get that

$$
\left\|\delta_{u}\right\|_{K} \leq \frac{C_{W}^{2}}{C_{\alpha}}\left(h_{K}\left\|\left(\boldsymbol{I} \boldsymbol{d}-\boldsymbol{P}_{\widetilde{W}}\right) \nabla \cdot \boldsymbol{q}\right\|_{K}+h_{K}^{1 / 2} R_{W^{\perp}}\|\alpha\|_{\partial K}\left\|I_{u}\right\|_{\partial K}\right) .
$$

The estimate of $\left\|\Pi_{W} u-u\right\|_{K}$ now follows by using the triangle inequality and by noting that $\left\|I_{u}\right\|_{F} \leq\left\|u-P_{W} u\right\|_{F}$ for any face $F$ of $K$ since, by the inclusion property (3.2b), we have that $I_{u}=P_{M} u-P_{W} u=P_{M}\left(u-P_{W} u\right)$.

It remains to prove the claim. By equation (3.10c), we have that

$$
\left\langle\boldsymbol{\delta}_{\boldsymbol{q}} \cdot \boldsymbol{n}+\alpha\left(\delta_{u}\right), w\right\rangle_{\partial K}=\left\langle\boldsymbol{I}_{\boldsymbol{q}} \cdot \boldsymbol{n}+\alpha\left(I_{u}\right), w\right\rangle_{\partial K} \quad \forall w \in W^{\perp}(K),
$$

because $\left.w\right|_{F} \in M(F)$ by the inclusion condition (3.2b).

But

$$
\left\langle\boldsymbol{\delta}_{\boldsymbol{q}} \cdot \boldsymbol{n}, w\right\rangle_{\partial K}=\left(\nabla \cdot \boldsymbol{\delta}_{\boldsymbol{q}}, w\right)_{K}+\left(\boldsymbol{\delta}_{\boldsymbol{q}}, \nabla w\right)_{K}=0 .
$$

Indeed, we have that $\left(\nabla \cdot \boldsymbol{\delta}_{\boldsymbol{q}}, w\right)_{K}=0$ by the first inclusion in condition (3.4b) and the fact that $w \in W^{\perp}(K)$. We also have that $\left(\boldsymbol{\delta}_{\boldsymbol{q}}, \nabla w\right)_{K}=0$ by equation (3.10a) and the first inclusion in condition (3.4a) . 
Similarly,

$$
\left\langle\boldsymbol{I}_{\boldsymbol{q}} \cdot \boldsymbol{n}, w\right\rangle_{\partial K}=\left(\nabla \cdot \boldsymbol{I}_{\boldsymbol{q}}, w\right)_{K}+\left(\boldsymbol{I}_{\boldsymbol{q}}, \nabla w\right)_{K}=\left(\left(\boldsymbol{I} \boldsymbol{d}-\boldsymbol{P}_{\widetilde{W}}\right) \nabla \cdot \boldsymbol{q}, w\right)_{K} .
$$

Indeed, $\left(\nabla \cdot \boldsymbol{I}_{\boldsymbol{q}}, w\right)_{K}=(\nabla \cdot \boldsymbol{q}, w)_{K}=\left(\left(\boldsymbol{I} \boldsymbol{d}-\boldsymbol{P}_{\widetilde{W}}\right) \nabla \cdot \boldsymbol{q}, w\right)_{K}$, by the first inclusion in condition (3.4b) and the fact that $w \in W^{\perp}(K)$. Moreover, $\left(\boldsymbol{I}_{\boldsymbol{q}}, \nabla w\right)_{K}=0$ by the first inclusion in condition (3.4a) and the definition of $\boldsymbol{I}_{\boldsymbol{q}}$. This proves the claim.

Step 3: The estimate of $\delta_{q}$. Finally, let us estimate of $\delta_{q}$. By the definition of $\boldsymbol{V}^{\perp}(K)$, 3.5a), we see that $\boldsymbol{\delta}_{\boldsymbol{q}} \in \boldsymbol{V}^{\perp}(K)$, by the equation (3.10a). By the condition (3.7a), this implies that

$$
\left\|\boldsymbol{\delta}_{\boldsymbol{q}}\right\|_{K} \leq C_{V} h_{K}^{1 / 2}\left\|\boldsymbol{\delta}_{\boldsymbol{q}} \cdot \boldsymbol{n}\right\|_{\partial K_{V}}
$$

and by equation (3.10c), that

$$
\left\|\boldsymbol{\delta}_{\boldsymbol{q}}\right\|_{K} \leq C_{V} h_{K}^{1 / 2}\left(\left\|\boldsymbol{I}_{\boldsymbol{q}} \cdot \boldsymbol{n}\right\|_{\partial K_{V}}+\left\|\alpha\left(\delta_{u}\right)\right\|_{\partial K_{V}}+\left\|\alpha\left(I_{u}\right)\right\|_{\partial K_{V}}\right) .
$$

If $\widetilde{W}(K)=W(K), \delta_{u}=0$, and we get that

$$
\left\|\boldsymbol{\delta}_{\boldsymbol{q}}\right\|_{K} \leq C_{V} h_{K}^{1 / 2}\left(\left\|\boldsymbol{I}_{\boldsymbol{q}} \cdot \boldsymbol{n}\right\|_{\partial K_{V}}+\|\alpha\|_{\partial K_{V}}\left\|I_{u}\right\|_{\partial K_{V}}\right) .
$$

If $\widetilde{W}(K) \neq W(K)$, then

$$
\left\|\boldsymbol{\delta}_{\boldsymbol{q}}\right\|_{K} \leq C_{V} h_{K}^{1 / 2}\left(\left\|\boldsymbol{I}_{\boldsymbol{q}} \cdot \boldsymbol{n}\right\|_{\partial K_{V}}+\|\alpha\|_{\partial K_{V}}\left(C_{W} R_{W^{\perp}}\left\|\delta_{u}\right\|_{\partial K_{W}}+\left\|I_{u}\right\|_{\partial K_{V}}\right)\right),
$$

by condition (3.7b). The estimate of $\left\|\boldsymbol{\Pi}_{V} \boldsymbol{q}-\boldsymbol{q}\right\|_{K}$ follows after using the triangle inequality and inserting the estimate of $\left\|\delta_{u}\right\|_{\partial K_{W}}$. This completes the proof of Theorem 3.1 .

\section{EXAMPles OF SUPERCONVERgENT METHODS}

In this section, we use the template described in the previous section to construct superconvergent HDG methods. We begin by discussing the three main examples of stabilization operator $\alpha$. We then give many examples of superconvergent methods using simplexes, squares, cubes and prisms. They include old and new (hybridized versions of) mixed and HDG methods. Finally, we end by briefly discussing how we used the template described in the previous section to construct the new superconvergent methods.

The verification of the inclusion properties in Steps 1 to 3 is fairly simple and will be left to the reader. However, for the most important cases, we are going to carry out the verification of the dimension count (3.6) and of the estimates (3.7) for the auxiliary spaces $\boldsymbol{V}^{\perp} \times W^{\perp}(K)$. To carry out the latter task, we only need to show that

$$
\begin{array}{ll}
\boldsymbol{v} \in \boldsymbol{V}^{\perp}(K) \text { and }\left.\boldsymbol{v} \cdot \boldsymbol{n}\right|_{\partial K_{V}}=0 & \text { implies } \boldsymbol{v}=0 \text { on } K, \\
w \in W^{\perp}(K) \text { and }\left.w\right|_{\partial K_{W}}=0 & \text { implies } w=0 \text { on } K .
\end{array}
$$

Indeed, the above conditions guarantee that $\|\boldsymbol{v} \cdot \boldsymbol{n}\|_{\partial K_{V}},\|w\|_{\partial K_{W}}$ define norms on $\boldsymbol{V}^{\perp}(K)$ and $W^{\perp}(K)$, respectively. The estimates (3.7) then follow by the finite dimensionality of the spaces under consideration and by standard scaling arguments.

4.1. The main local stabilization operators $\alpha$. There are three main examples of local stabilization operators $\alpha$ satisfying conditions (3.8). 
(1) No stabilization. The first example is the trivial choice $\alpha:=0$ which is used in all the mixed methods.

(2) Pointwise stabilization. The second example is $\alpha:=\tau I d$. If $\tau$ is taken to be a non-negative constant on each face $F$ of each of the elements $K \in \mathcal{T}_{h}$ and strictly positive on one arbitrary face $F_{K} \in \partial K$, it is very easy to see that this operator satisfies the condition (3.8a) of being self-adjoint as well as the coercivity condition (3.8b) with $C_{\alpha}:=\left.\tau\right|_{F_{K}}$ and $\partial K_{W}:=F_{K}$. Finally, note that $\|\alpha\|_{F}=\left.\tau\right|_{F}$.

(3) Averaging stabilization. The third and last example is the local stabilization operator used by the so-called BMMPR-H methods; see also 10] and the references therein. It is given by $\alpha:=\tau \boldsymbol{r} \cdot \boldsymbol{n}$, where $\boldsymbol{r}$ is a suitably defined lifting operator. In [3] and [7] (see also 2]) such operator was introduced in the case in which $K$ is a simplex and $\boldsymbol{V}(K) \times W(K):=\boldsymbol{P}^{k}(K) \times P^{k}(K)$ and $M(F):=P^{k}(F)$. We can extend its definition as follows. Given an element $K$, for any $\mu \in M(F)$, we define $\boldsymbol{r}(\mu)$ on $K$ as the element of $\boldsymbol{V}(K)$ satisfying

$$
\frac{1}{|K|}(\boldsymbol{r}(\mu), \boldsymbol{v})_{K}=\frac{1}{|F|}\langle\mu, \boldsymbol{v} \cdot \boldsymbol{n}\rangle_{F} \quad \text { for all } \boldsymbol{v} \in \boldsymbol{V}(K) .
$$

It is not difficult to see that the operator $\alpha$ satisfies the condition of being selfadjoint (3.8a) since, by taking $\boldsymbol{v}:=\alpha(\eta) \boldsymbol{n}$ in the definition of $\boldsymbol{r}$, we get

$$
\langle\alpha(\eta), \mu\rangle_{F}=\tau_{F}\langle\boldsymbol{r}(\eta) \cdot \boldsymbol{n}, \mu\rangle_{F}=\tau_{F} \frac{|F|}{|K|}(\boldsymbol{r}(\eta), \boldsymbol{r}(\mu))_{K} .
$$

Unlike the previous example, the verification of the positivity condition (3.8b depends on the choice of local spaces. It is guaranteed if, for any $w^{\perp} \in W^{\perp}(K)$, we can find an element $\boldsymbol{v}$ of $\boldsymbol{V}(K)$ such that

$$
\forall w^{\perp} \in W^{\perp}(K) \exists \boldsymbol{v} \in \boldsymbol{V}(K):\left.\boldsymbol{v} \cdot \boldsymbol{n}\right|_{F}=\left.w^{\perp}\right|_{F},\|\boldsymbol{v}\|_{K} \leq \mathrm{C} \frac{|K|^{1 / 2}}{|F|^{1 / 2}}\left\|w^{\perp}\right\|_{F} .
$$

Indeed, we have that

$$
\left\|w^{\perp}\right\|_{F}^{2}=\left\langle w^{\perp}, \boldsymbol{v} \cdot \boldsymbol{n}\right\rangle_{F}=\frac{|F|}{|K|}\left(\boldsymbol{r}\left(w^{\perp}\right), \boldsymbol{v}\right)_{K} \leq \mathrm{C} \frac{|F|^{1 / 2}}{|K|^{1 / 2}}\left\|\boldsymbol{r}\left(w^{\perp}\right)\right\|_{K}\left\|w^{\perp}\right\|_{F},
$$

and so $\left\|w^{\perp}\right\|_{F} \leq \mathrm{C} \frac{|F|^{1 / 2}}{|K|^{1 / 2}}\left\|\boldsymbol{r}\left(w^{\perp}\right)\right\|_{K}$. On the other hand,

$$
\left\langle\alpha\left(w^{\perp}\right), w^{\perp}\right\rangle_{F}=\tau \frac{|F|}{|K|}\left\|\boldsymbol{r}\left(w^{\perp}\right)\right\|^{2} \geq \frac{\tau}{\mathrm{C}^{2}}\left\|w^{\perp}\right\|_{F}^{2} .
$$

We thus see that the coercivity condition $3.8 \mathrm{~b}$ is satisfied with $C_{\alpha}:=\tau / \mathrm{C}^{2}$ and $\partial K_{W}:=F$.

It turns out that the condition (4.2) is verified for all the examples we present in this paper.

4.2. Methods using simplexes. We begin by considering methods for which the element $K$ is a simplex. 
4.2.1. Description of the methods. To describe the methods, we use the following notation: $P^{k}(D)$ denotes the space of polynomials of total degree $k$ defined on $D$, $\widetilde{P}^{k}(D)$ denotes the space of homogeneous polynomials of degree $k$ defined on $D$, $\boldsymbol{P}^{k}(D)$ denotes the space $\left[P^{k}(D)\right]^{n}, \mathcal{R}^{k}(\partial K)$ denotes the functions whose restriction to each face $F$ of $K$ belong to $P^{k}(F)$, and $\boldsymbol{\Phi}_{k}(K)$ denotes the space of functions in $\boldsymbol{P}^{k}(K)$ which are divergence-free and whose normal component on $\partial K$ is zero.

TABle 1. Methods for which $M(F)=P^{k}(F), k \geq 1$, and $K$ is a simplex.

\begin{tabular}{ccccc}
\hline method & $\boldsymbol{V}(K)$ & $W(K)$ & $\widetilde{\boldsymbol{V}}(K)$ & $\widetilde{W}(K)$ \\
\hline \hline $\mathbf{B D F M}_{k+1}$ & $\left\{\boldsymbol{q} \in \boldsymbol{P}^{k+1}(K):\right.$ & $P^{k}(K)$ & $\nabla P^{k}(K) \oplus \boldsymbol{\Phi}_{k+1}(K)$ & $P^{k}(K)$ \\
& $\left.\left.\boldsymbol{q} \cdot \boldsymbol{n}\right|_{\partial K} \in \mathcal{R}^{k}(\partial K)\right\}$ & & & \\
$\mathbf{R T}_{k}$ & $\boldsymbol{P}^{k}(K) \oplus \boldsymbol{x} \widetilde{P}^{k}(K)$ & $P^{k}(K)$ & $\boldsymbol{P}^{k-1}(K)$ & $P^{k}(K)$ \\
$\mathbf{H D G}_{k}$ & $\boldsymbol{P}^{k}(K)$ & $P^{k}(K)$ & $\boldsymbol{P}^{k-1}(K)$ & $P^{k-1}(K)$ \\
$\mathbf{B D M}_{k \geq 2}$ & $\boldsymbol{P}^{k}(K)$ & $P^{k-1}(K)$ & $\nabla P^{k-1}(K) \oplus \boldsymbol{\Phi}_{k}(K)$ & $P^{k-1}(K)$ \\
\hline
\end{tabular}

TABLE 2. Orders of convergence for methods for which $M(F)=$ $P^{k}(F), k \geq 1$, and $K$ is a simplex.

\begin{tabular}{ccccccc}
\hline method & $\partial K_{V}$ & $\partial K_{W}$ & $\tau$ & $\left\|\boldsymbol{q}-\boldsymbol{q}_{h}\right\|_{\mathcal{T}_{h}}\left\|\Pi_{W} u-u_{h}\right\|_{\mathcal{T}_{h}}\left\|u-u_{h}^{\star}\right\|_{\mathcal{T}_{h}}$ \\
\hline \hline $\mathbf{B D F M}_{k+1}$ & $\partial K$ & - & 0 & $k+1$ & $k+2$ & $k+2$ \\
$\mathbf{R T}_{k}$ & $\partial K$ & - & 0 & $k+1$ & $k+2$ & $k+2$ \\
$\mathbf{H D G}_{k}$ & $\partial K \backslash F_{K}$ & $F_{K}$ & $\mathcal{O}(1),>0$ & $k+1$ & $k+2$ & $k+2$ \\
$\mathbf{B D M}_{k}$ & $\partial K$ & - & 0 & $k+1$ & $k+2$ & $k+2$ \\
$k \geq 2$ & & & & & & \\
\hline
\end{tabular}

In Tables 1 and2, we give methods that satisfy all the conditions in Steps 1 to 4 in our template. The orders of convergence, for smooth solutions, follow by combining our main results with the approximation properties of the auxiliary projection given by Theorem 3.1

The methods are the well-known mixed methods of Raviart-Thomas, $\mathbf{R T}_{k}$, of Brezzi-Douglas-Marini, $\mathbf{B D M}_{k}$, and of Brezzi-Douglas-Fortin-Marini, $\mathbf{B D F M}_{k+1}$. In the three-dimensional case, the $\mathbf{R T}_{k}$ method was introduced in [15, and the method $\mathbf{B D M}_{k}$ in [4. The $\mathbf{H D G}_{k}$ method with $\alpha:=\tau I d$ was proposed in [10; since the condition (4.2) is satisfied, we can also use $\alpha:=\tau \boldsymbol{r} \cdot \boldsymbol{n}$.

4.2.2. Verification of the conditions on $\boldsymbol{V}^{\perp}(K) \times W^{\perp}(K)$. The projections $\Pi_{h}$ associated with the mixed methods can be also constructed as indicated by our template; see [6]. In particular, the property (4.1a) is satisfied with $\partial K_{V}=\partial K$ and, since $W^{\perp}(K)=\emptyset$ (and $\alpha \equiv 0$ ), the property (4.1b) is automatically satisfied for any $\partial K_{W} \subset \partial K$. The same remark can be made about the projection $\Pi_{h}$ for the $\mathbf{H D G}_{k}$ method proposed in [11.

For the sake of completeness, let us verify that the conditions on the local space $\boldsymbol{V}^{\perp}(K) \times W^{\perp}(K)$ of the $\mathbf{H D G}_{k}$ method are satisfied. We begin by verifying the 
dimension count (3.6):

$$
\begin{aligned}
\sum_{F \in \mathcal{F}(K)} \operatorname{dim} M(F) & =(d+1) \operatorname{dim} P_{k}(F), \\
\operatorname{dim} \boldsymbol{V}^{\perp}(K) & =\operatorname{dim} \boldsymbol{V}(K)-\operatorname{dim} \widetilde{\boldsymbol{V}}(K) \\
& =d\left(\operatorname{dim} P_{k}(K)-\operatorname{dim} P_{k-1}(K)\right) \\
& =d \operatorname{dim} P_{k}(F), \\
\operatorname{dim} W^{\perp}(K) & =\operatorname{dim} W(K)-\operatorname{dim} \widetilde{W}(K) \\
& =\operatorname{dim} P_{k}(K)-\operatorname{dim} P_{k-1}(K) \\
& =\operatorname{dim} P_{k}(F),
\end{aligned}
$$

and the condition (3.6) follows.

Let us now verify the estimates (3.7). Take $w \in W^{\perp}(K)$ such that $\left.w\right|_{F_{K}}=0$. Then, we can write that $w=\lambda_{F_{K}} w^{\prime}$, where $w^{\prime} \in P^{k-1}(K)$ and $\lambda_{F_{K}}$ is the barycentric coordinate function associated with the vertex of the simplex $K$ opposite to the face $F_{K}$. Since $w \in W^{\perp}(K)$,

$$
0=\left(w, w^{\prime}\right)_{K}=\left(\lambda_{F_{K}} w^{\prime}, w^{\prime}\right)_{K}
$$

and this implies that $w^{\prime}=0$ on $K$ since $\lambda_{F_{K}}$ is always positive on $K$. This shows that (4.1b) holds.

We can verify the estimate (4.1a) in a similar way. Given any $\boldsymbol{v} \in \boldsymbol{V}^{\perp}(K)$, we can apply the above argument for each $\boldsymbol{v} \cdot \boldsymbol{n}_{F}, F \in \partial K \backslash F_{K}$, to conclude that $\boldsymbol{v} \cdot \boldsymbol{n}_{F}=0$ in $K$. Since $\left\{\boldsymbol{n}_{F}: F \in \partial K \backslash F_{K}\right\}$ is a basis of $\mathbb{R}^{d}$, we have that $\boldsymbol{v}=\mathbf{0}$.

4.3. Methods using squares and cubes with $M(F)=P^{k}(F)$. Next, we consider methods for which the element $K$ is a square $(n=2)$ or a cube $(n=3)$ and for which the space $M(F)$ is $P^{k}(F)$.

4.3.1. Description of the methods. Here, $P^{\ell_{1}, \ell_{2}}(D)$ for $n=2$ and $P^{\ell_{1}, \ell_{2}, \ell_{3}}$ for $n=3$ denote the space of polynomials of degree $\ell_{i}$ on the $i$-th variable, $i=1, \ldots, n$.

In Table 3. we display the methods using squares and in Table 4. those using cubes. In Table 5, we display their orders of convergence. The mixed methods $\mathbf{B D F M}_{[k+1]}$ and $\mathbf{B D M}_{[k]}$ are well known but the $\mathbf{H D G}_{[k]}^{P}$ method is new.

TABLE 3. Methods for which $M(F)=P^{k}(F), k \geq 1$, and $K$ is a square.

\begin{tabular}{ccccc}
\hline method & $\boldsymbol{V}(K)$ & $W(K)$ & $\widetilde{\boldsymbol{V}}(K)$ & $\widetilde{W}(K)$ \\
\hline \hline $\mathbf{B D F M}_{[k+1]}$ & $P^{k+1}(K) \backslash\left\{y^{k+1}\right\}$ & $P^{k}(K)$ & $\boldsymbol{P}^{k-1}(K)$ & $P^{k}(K)$ \\
$\mathbf{H D G}_{[k]}^{P}$ & $\times\left(P^{k+1}(K) \backslash\left\{x^{k+1}\right\}\right)$ & & & \\
& $\boldsymbol{P}^{k}(K)$ & $P^{k}(K)$ & $\boldsymbol{P}^{k-1}(K)$ & $P^{k-1}(K)$ \\
$\mathbf{B D M}_{[k]}$ & $\oplus \nabla \times\left(x y \widetilde{P}^{k}(K)\right)$ & & & \\
$k \geq 2$ & $\boldsymbol{P}^{k}(K)$ & $P^{k-1}(K)$ & $\boldsymbol{P}^{k-2}(K)$ & $P^{k-1}(K)$ \\
& $\oplus \nabla \times\left(x y x^{k}\right)$ & & & \\
\hline
\end{tabular}


TABle 4. Methods for which $M(F)=P^{k}(F), k \geq 1$, and $K$ is a cube.

\begin{tabular}{ccccc}
\hline method & $\boldsymbol{V}(K)$ & $W(K)$ & $\widetilde{\boldsymbol{V}}(K)$ & $\widetilde{W}(K)$ \\
\hline \hline $\mathbf{B D F M}_{[k+1]}$ & $P^{k+1}(K) \backslash \widetilde{P}^{k+1}(y, z)$ & $P^{k}(K)$ & $\boldsymbol{P}^{k-1}$ & $P^{k}(K)$ \\
& $\times P^{k+1}(K) \backslash \widetilde{P}^{k+1}(x, z)$ & & & \\
& $\times P^{k+1}(K) \backslash \widetilde{P}^{k+1}(x, y)$ & & & \\
$\mathbf{H D G}_{[k]}^{P}$ & $\boldsymbol{P}^{k}(K)$ & $P^{k}(K)$ & $\boldsymbol{P}^{k-1}(K)$ & $P^{k-1}(K)$ \\
& $\oplus \nabla \times\left(y z \widetilde{P}^{k}(K), 0,0\right)$ & & & \\
& $\oplus \nabla \times\left(0, z x \widetilde{P}^{k}(K), 0\right)$ & & & \\
$\mathbf{B D M}_{[k]}$ & $\boldsymbol{P}^{k}(K)$ & $P^{k-1}(K)$ & $\boldsymbol{P}^{k-2}(K)$ & $P^{k-1}(K)$ \\
$k \geq 2$ & $\oplus \nabla \times\left(0,0, x y \widetilde{P}^{k}(y, z)\right)$ & & & \\
& $\oplus \nabla \times\left(0, x z \widetilde{P}^{k}(x, y), 0\right)$ & & & \\
& $\oplus \nabla \times\left(y z \widetilde{P}^{k}(x, z), 0,0\right)$ & & & \\
\hline
\end{tabular}

TABLE 5. Orders of convergence for methods for which $M(F)=$ $P^{k}(F), k \geq 1$, and $K$ is a square or a cube.

\begin{tabular}{ccccccc}
\hline method & $\partial K_{V} \partial K_{W}$ & $\tau$ & \multicolumn{4}{c}{$\left\|\boldsymbol{q}-\boldsymbol{q}_{h}\right\|_{\mathcal{T}_{h}}\left\|\Pi_{W} u-u_{h}\right\|_{\mathcal{T}_{h}}\left\|u-u_{h}^{\star}\right\|_{\mathcal{T}_{h}}$} \\
\hline \hline $\mathbf{B D F M}_{[k+1]}$ & $\partial K$ & - & 0 & $k+1$ & $k+2$ & $k+2$ \\
$\mathbf{H D G}_{[k]}^{P}$ & $\partial K$ & $F_{K}$ & $\mathcal{O}(1),>0$ & $k+1$ & $k+2$ & $k+2$ \\
$\mathbf{B D M}_{[k]}$ & $\partial K$ & - & 0 & $k+1$ & $k+2$ & $k+2$ \\
$k \geq 2$ & & & & & \\
\hline
\end{tabular}

4.3.2. Verification of the conditions on $\boldsymbol{V}^{\perp}(K) \times W^{\perp}(K)$. For the mixed methods; see [6]. Let us now consider the $\mathbf{H D G}_{[k]}^{P}$ method for the case in which $K$ is a cube; the case for which $K$ is a square is simpler.

We begin by verifying the dimension count (3.6):

$$
\begin{aligned}
\sum_{F \in \mathcal{F}(K)} \operatorname{dim} M(F) & =6 \operatorname{dim} P_{k}(F), \\
\operatorname{dim} \boldsymbol{V}^{\perp}(K) & =\operatorname{dim} \boldsymbol{V}(K)-\operatorname{dim} \widetilde{\boldsymbol{V}}(K) \\
& \left.=\left(3 \operatorname{dim} P_{k}(K)+2 \operatorname{dim} \widetilde{P}_{k}(K)\right)-3 \operatorname{dim} P_{k-1}(K)\right) \\
& =5 \operatorname{dim} P_{k}(F), \\
\operatorname{dim} W^{\perp}(K) & =\operatorname{dim} W(K)-\operatorname{dim} \widetilde{W}(K) \\
& =\operatorname{dim} P_{k}(K)-\operatorname{dim} P_{k-1}(K) \\
& =\operatorname{dim} P_{k}(F),
\end{aligned}
$$

and the condition (3.6) follows.

The proof for (4.1b) is exactly the same as the proof in the simplex case since the spaces $W(K)$ and $\widetilde{W}(K)$ are the same. Let us verify 4.1a). Take $\boldsymbol{v} \in \boldsymbol{V}^{\perp}(K)$ and assume that $\boldsymbol{v} \cdot \boldsymbol{n}=0$ on $\partial K$. If two parallel faces of the cube $K$ lie on the the planes $x=a, x=b$, respectively, we can conclude that $v_{1}=0$ on $x=a, x=b$, where $v_{1}$ is the first component of $\boldsymbol{v}$. So we can write $v_{1}=(x-a)(x-b) v_{1}^{\prime}$ where 
$v_{1}^{\prime} \in P^{k-1}(K)$. Since $\boldsymbol{v} \in \boldsymbol{V}^{\perp}(K)$ and $\boldsymbol{v}^{\prime}:=\left(v_{1}^{\prime}, 0,0\right) \in \tilde{\boldsymbol{V}}(K)$, we have that

$$
0=\left(\boldsymbol{v}, \boldsymbol{v}^{\prime}\right)_{K}=\left((x-a)(x-b) v_{1}^{\prime}, v_{1}^{\prime}\right)_{K}
$$

and we can conclude that $v_{1}^{\prime}=0$ on $K$. This implies that $v_{1}=0$ on $K$. A similar argument can be applied for the other two components. This means that $\boldsymbol{v}=\mathbf{0}$ on $K$.

4.4. Methods using squares and cubes with $M(F)=Q^{k}(F)$. Next, we consider methods for which the element $K$ is a square $(n=2)$ or a cube $(n=3)$ and for which the space $M(F)$ is $Q^{k}(F)$. The main motivation for exploring this type of methods is that their tensor-product structure can be exploited to achieve a very efficient implementation; see [20, 19, 8] and the references therein.

4.4.1. Description of the methods. To describe the methods, we use the following notation. We denote by $Q^{k}(D)$ the space of polynomials of degree $k$ in each variable defined on $D$, and by $\boldsymbol{Q}^{k}(D)$ the space $\left[Q^{k}(D)\right]^{n}$. We also set

$$
\begin{aligned}
V_{0}(K):= & \left\{\boldsymbol{v} \in \boldsymbol{V}(K):\left.\boldsymbol{v} \cdot \boldsymbol{n}\right|_{\partial K}=0\right\}, \\
\boldsymbol{S}^{k}(K):= & \left\{\boldsymbol{v} \in \boldsymbol{V}_{0}(K): \nabla \cdot \boldsymbol{v}=0\right\}, \\
\boldsymbol{H}^{k}(K):= & \left\{\left(\left(x^{2}-x\right) x^{k-1}\left(a L_{k}(y)+b\right),\left(y^{2}-y\right) y^{k-1}\left(c L_{k}(z)+d\right),\right.\right. \\
& \left.\left.\left(\left(z^{2}-z\right) z^{k-1}\left(e L_{k}(x)+f\right)\right)\right):(a, b, c, d, e, f) \in \mathbb{R}^{6}\right\}, \\
\boldsymbol{H}_{M}^{k}(K):= & \boldsymbol{H}^{k}(K) \oplus\left\{\left(\left(x^{2}-x\right) x^{k-1} L_{k}(y) L_{k}(z), 0,0\right)\right\},
\end{aligned}
$$

here $L_{i}(x)$ denotes the scaled Legendre polynomial of degree $i$ on the interval $[0,1]$.

In Table [6] we display the methods using squares and in Table 7 , those using cubes. Without lost of generality, we present the spaces on the reference domain $K=[0,1]^{3}$. In Table 8 , we present their orders of convergence.

The mixed method $\mathbf{R} \mathbf{T}_{[k]}$ is well known but the methods $\mathbf{T N} \mathbf{T}_{[k]}$ and $\mathbf{H D G}_{[k]}^{Q}$ are new. As we see in Table 8 these three methods achieve the same orders of convergence. However, as pointed out in the Introduction, the spaces of the new methods are significantly smaller than those of the $\mathbf{R} \mathbf{T}_{[k]}$ method. For example, in the case of cubic elements, only by adding 7 or 6 new basis functions to the space $\boldsymbol{Q}^{k}(K)$ we obtain the superconvergent methods $\mathbf{T} \mathbf{N} \mathbf{T}_{[k]}$ and $\mathbf{H D G}_{[k]}^{Q}$, respectively. Note that the method $\mathbf{T N T}_{[k]}$ (whose name stems from the fact that its local space $\boldsymbol{V}(K)$ is a tiny space containing the tensor product space $\boldsymbol{Q}^{k}(K)$ ) is a mixed method, as its stabilization function can be taken to be identically zero.

TABLE 6 . Methods for which $M(F)=Q^{k}(F), k \geq 1$, and $K$ is a square.

\begin{tabular}{ccccc}
\hline method & $\boldsymbol{V}(K)$ & $W(K)$ & $\widetilde{\boldsymbol{V}}(K)$ & $\widetilde{W}(K)$ \\
\hline \hline $\mathbf{R T}_{[k]}$ & $P^{k+1, k}(K)$ & $Q^{k}(K)$ & $P^{k-1, k}(K)$ & $Q^{k}(K)$ \\
& $\times P^{k, k+1}(K)$ & & $\times P^{k, k-1}(K)$ & \\
$\mathbf{T N T}_{[k]}$ & $\boldsymbol{Q}^{k}(K)$ & $Q^{k}(K)$ & $\nabla Q^{k}(K) \oplus \boldsymbol{S}^{k}(K)$ & $Q^{k}(K)$ \\
& $\oplus\left\{\left(x^{k+1}, 0\right),\left(0, y^{k+1}\right)\right\}$ & & & \\
$\mathbf{H D G}_{[k]}^{Q}$ & $\oplus\left\{\left(x^{k+1} y^{k}, 0\right)\right\}$ & & & \\
& $\oplus\left\{\left(x^{k+1}, 0\right),\left(0, y^{k+1}\right)\right\}$ & & & \\
& & & & \\
& & & & \\
\end{tabular}


TABLE 7. Methods for which $M(F)=Q^{k}(F), k \geq 1$, and $K$ is a cube.

\begin{tabular}{ccccc}
\hline method & $\boldsymbol{V}(K)$ & $W(K)$ & $\widetilde{\boldsymbol{V}}(K)$ & $\widetilde{W}(K)$ \\
\hline \hline $\mathbf{R T}_{[k]}$ & $P^{k+1, k, k}(K)$ & $Q^{k}(K)$ & $P^{k-1, k, k}(K)$ & $Q^{k}(K)$ \\
& $\times P^{k, k+1, k}(K)$ & & $\times P^{k, k-1, k}(K)$ & \\
& $\times P^{k, k, k+1}(K)$ & & $\times P^{k, k, k-1}(K)$ & \\
$\mathbf{T N T}_{[k]}$ & $\boldsymbol{Q}^{k}(K) \oplus \boldsymbol{H}_{M}^{k}(K)$ & $Q^{k}(K)$ & $\nabla Q^{k}(K) \oplus \boldsymbol{S}_{k}(K)$ & $Q^{k}(K)$ \\
$\mathbf{H D G}_{[k]}^{Q}$ & $\boldsymbol{Q}^{k}(K) \oplus \boldsymbol{H}^{k}(K)$ & $Q^{k}(K)$ & $\nabla Q^{k}(K) \oplus \boldsymbol{S}_{k}(K)$ & $Q^{k}(K) \backslash\left\{x^{k} y^{k} z^{k}\right\}$ \\
\hline
\end{tabular}

TABLE 8. Orders of convergence for methods for which $M(F)=$ $Q^{k}(F), k \geq 1$, and $K$ is a square or a cube.

\begin{tabular}{ccccccc}
\hline $\operatorname{method}$ & $\partial K_{V} \partial K_{W}$ & $\tau$ & \multicolumn{4}{c}{$\left\|\boldsymbol{q}_{h}\right\|_{\mathcal{T}_{h}}\left\|\Pi_{W} u-u_{h}\right\|_{\mathcal{T}_{h}}\left\|u-u_{h}^{\star}\right\|_{\mathcal{T}_{h}}$} \\
\hline \hline $\mathbf{R T}_{[k+1]}$ & $\partial K$ & - & 0 & $k+1$ & $k+2$ & $k+2$ \\
$\mathbf{T N T}_{[k]}$ & $\partial K$ & - & 0 & $k+1$ & $k+2$ & $k+2$ \\
$\mathbf{H D G}_{[k]}^{Q}$ & $\partial K$ & $F_{K}$ & $\mathcal{O}(1)>0$ & $k+1$ & $k+2$ & $k+2$ \\
\hline
\end{tabular}

4.4.2. Verification of the conditions on $\boldsymbol{V}^{\perp}(K) \times W^{\perp}(K)$. Again for the mixed method, see [6]. We only consider the method $\mathbf{H D G}_{[k]}^{Q}$ for cubic elements since the verification of the conditions on $\boldsymbol{V}^{\perp}(K) \times W^{\perp}(K)$ for squares is much simpler. The verification of those properties for the method $\mathbf{T N T}_{[k]}$ is almost identical.

We begin by verifying the dimension count (3.6). To do that, we first need to study the space $\widetilde{\boldsymbol{V}}(K)=\nabla Q^{k}(K) \oplus \boldsymbol{S}^{k}(K)$.

Lemma 4.1. We have that $\nabla Q^{k}(K) \oplus \boldsymbol{S}^{k}(K)$ is a direct sum.

Proof. Let us show that $\nabla Q^{k}(K) \cap \boldsymbol{S}^{k}(K)=\emptyset$. Assume that there is a non-zero function $\boldsymbol{v}$ in $\nabla Q^{k}(K) \cap \boldsymbol{S}^{k}(K)$. Since $\boldsymbol{v}$ in $\nabla Q^{k}(K)$, there is $w \in Q^{k}(K)$ such that $\boldsymbol{v}=\nabla w$. As a consequence,

$$
\|\boldsymbol{v}\|_{K}^{2}=(\nabla w, \boldsymbol{v})_{K}=-(w, \nabla \cdot \boldsymbol{v})+\langle\boldsymbol{v} \cdot \boldsymbol{n}, w\rangle_{\partial K}=0,
$$

since $\boldsymbol{v} \in \boldsymbol{S}^{k}(K)$. This implies that $\boldsymbol{v}=\mathbf{0}$ and completes the proof.

The second lemma gives the dimension of $\boldsymbol{S}^{k}(K)$.

Lemma 4.2. We have

$$
\operatorname{dim} \boldsymbol{S}^{k}(K)=2 \operatorname{dim} Q^{k}(K)-6 \operatorname{dim} Q^{k}(F)+8 .
$$

Proof. By definition of the space $\boldsymbol{S}^{k}(K)$, we have that

$$
\operatorname{dim} \boldsymbol{S}^{k}=\operatorname{dim} \boldsymbol{V}_{0}(K)-\operatorname{dim} \nabla \cdot \boldsymbol{V}_{0}(K) .
$$

Now, by definition of the space $\boldsymbol{V}_{0}(K)$, we can write that $\boldsymbol{V}_{0}(K)=\boldsymbol{E}_{0}(K) \oplus$ $\boldsymbol{H}^{k}(K)$, where

$$
\begin{aligned}
\boldsymbol{E}_{0}(K): & :=\left\{\boldsymbol{v} \in \boldsymbol{Q}^{k}(K):\left.\boldsymbol{v} \cdot \boldsymbol{n}\right|_{\partial K}=0\right\} \\
& =\left(x^{2}-x\right) P^{k-2, k, k}(K) \times\left(y^{2}-y\right) P^{k, k-2, k}(K) \times\left(z^{2}-z\right) P^{k, k, k-2}(K) .
\end{aligned}
$$


Next, we consider the spaces $\nabla \cdot \boldsymbol{E}_{0}(K)$ and $\nabla \cdot \boldsymbol{H}^{k}(K)$. Note that any function $f \in\left(x^{2}-x\right) P^{k-2}(0,1)$ must be of the form

$$
f(x)=\sum_{\ell=1}^{k-1} a_{\ell} \int_{0}^{x} L_{\ell}(s) d s .
$$

This means that we can write

$$
\begin{aligned}
\nabla \cdot \boldsymbol{E}_{0}(K)=\operatorname{span}\left\{L_{i_{1}}(x) L_{i_{2}}(y) L_{i_{3}}(z):\right. & 1 \leq i_{1} \leq k-1,0 \leq i_{2}, i_{3} \leq k \quad \text { or } \\
& 1 \leq i_{2} \leq k-1,0 \leq i_{1}, i_{3} \leq k \quad \text { or } \\
& \left.1 \leq i_{3} \leq k-1,0 \leq i_{1}, i_{2} \leq k\right\} .
\end{aligned}
$$

On the other hand, we have that

$$
\begin{aligned}
\nabla \cdot \boldsymbol{H}^{k}(K)=\operatorname{span}\{ & \left((k+1) x^{k}-k x^{k-1}\right) L_{k}(y),(k+1) x^{k}-k x^{k-1}, \\
& \left((k+1) y^{k}-k y^{k-1}\right) L_{k}(z),(k+1) y^{k}-k y^{k-1}, \\
& \left.\left((k+1) z^{k}-k z^{k-1}\right) L_{k}(x),(k+1) z^{k}-k z^{k-1}\right\},
\end{aligned}
$$

and we can see that the basis functions in $\nabla \cdot \boldsymbol{H}^{k}(K)$ are linearly independent with the basis functions of $\nabla \cdot \boldsymbol{E}_{0}(K)$.

This implies that

$$
\begin{aligned}
\operatorname{dim} \boldsymbol{S}^{k}(K) & =\operatorname{dim} \boldsymbol{E}_{0}(K)-\operatorname{dim} \nabla \cdot \boldsymbol{E}_{0}(K) \\
& =\left(3 \operatorname{dim} Q^{k}(K)-6 \operatorname{dim} Q^{k}(F)\right)-\left(\operatorname{dim} Q^{k}(K)-8\right) .
\end{aligned}
$$

This completes the proof.

We are now ready to verify the dimension count (3.6). We have

$$
\begin{aligned}
\sum_{F \in \mathcal{F}(K)} \operatorname{dim} M(F) & =6 \operatorname{dim} Q^{k}(F), \\
\operatorname{dim} W^{\perp}(K) & =\operatorname{dim} W(K)-\operatorname{dim} \widetilde{W}(K)=1,
\end{aligned}
$$

and, by Lemma 4.1

$$
\begin{aligned}
\operatorname{dim} \boldsymbol{V}^{\perp}(K) & =\operatorname{dim} \boldsymbol{V}(K)-\operatorname{dim} \tilde{\boldsymbol{V}}(K) \\
& =\left(3 \operatorname{dim} Q^{k}(K)+6\right)-\left(\operatorname{dim} \nabla Q^{k}(K)+\operatorname{dim} \boldsymbol{S}^{k}(K)\right) \\
& =2 \operatorname{dim} Q^{k}(K)+7-\operatorname{dim} \boldsymbol{S}^{k}(K) \\
& =6 \operatorname{dim} Q^{k}(F)-1,
\end{aligned}
$$

by Lemma 4.2. This means that the condition (3.6) is verified.

Now, let us show that the properties (4.1) are true. Let us take $w \in W^{\perp}(K)$ such that $\left.w\right|_{F_{K}}=0$. Then we have that $w=\lambda_{F_{K}} w^{\prime}$, where $w^{\prime} \in \widetilde{W}(K)$. By using the same argument as in the proof in the case of simplex we can conclude that $w^{\prime}=0$ which in turn implies that $w=0$. So (4.1b) is true.

Now take $\boldsymbol{v} \in \boldsymbol{V}^{\perp}(K)$ such that $\left.\boldsymbol{v} \cdot \boldsymbol{n}\right|_{\partial K}=0$. We are going to show that $\boldsymbol{v} \in \boldsymbol{S}^{k}(K)$, which would imply that $\boldsymbol{v}=\mathbf{0}$ on $K$, since $\tilde{\boldsymbol{V}}(K) \supset \boldsymbol{S}^{k}(K)$. To do that, we note that, for any $w \in W(K)=Q^{k}(K)$,

$$
(\nabla \cdot \boldsymbol{v}, w)_{K}=\langle\boldsymbol{v} \cdot \boldsymbol{n}, w\rangle_{\partial K}-(\boldsymbol{v}, \nabla w)_{K}=0,
$$


because $\tilde{\boldsymbol{V}}(K) \supset \nabla Q^{k}(K)$. Since $\nabla \cdot \boldsymbol{V}(K) \subset W(K)=Q^{k}(K)$, this implies that $\nabla \cdot \boldsymbol{v}=0$ and $\boldsymbol{v} \in \boldsymbol{S}^{k}(K)$. This shows that (4.1a) is also true.

4.5. Methods using prisms. Finally, we present three prismatic finite elements.

4.5.1. Description of the methods. We consider the prism whose base is a triangle in the $(x, y)$-plane and whose lateral faces are parallel to the $z$-axis. We denote by $P^{m \mid n}(K)$ the space of polynomials of degree $m$ in the two variables $x$ and $y$ and of degree $n$ in the variable $z$. We also denote by $M_{V}(F), M_{H}(F)$ the finite dimensional spaces $M(F)$ on the vertical and horizontal faces, respectively. Finally, we set

$$
\begin{aligned}
B^{k+2}(K) & :=\left\{w \in P^{k+2}(K):\left.w\right|_{F}=0, \text { on three vertical faces }\right\}, \\
B^{k+2 \mid k}(K) & :=\left\{w \in P^{k+2 \mid k}(K):\left.w\right|_{F}=0, \text { on three vertical faces }\right\}, \\
\boldsymbol{Y}^{k+1}(K) & :=\left[\nabla_{(x, y)} P^{k-1}(K) \oplus \nabla_{(x, y)} \times B^{k+2}(K)\right] \times P^{k-1}(K), \\
\boldsymbol{Z}^{k}(K) & :=\left[\nabla_{(x, y)} P^{k \mid k}(K) \oplus \nabla_{(x, y)} \times B^{k+2 \mid k}(K)\right] \times P^{k \mid k-1}(F) .
\end{aligned}
$$

Here, $\nabla_{(x, y)}, \nabla_{(x, y)} \cdot \nabla_{(x, y)} \times$ denote the corresponding differential operators in the variables $x$ and $y$.

In Table 9, we display the methods and in Table 10, we present their orders of

\begin{tabular}{|c|c|c|c|c|c|}
\hline \multicolumn{6}{|c|}{$\mathrm{BDFM}_{\langle\mathrm{k}+1\rangle}$} \\
\hline $\boldsymbol{V}(K)$ & $W(K)$ & $\tilde{\boldsymbol{V}}(K)$ & $\widetilde{W}(K)$ & $M_{V}(F)$ & $M_{H}(F)$ \\
\hline $\begin{array}{c}P^{k+1}(K) \backslash\left\{z^{k+1}\right\} \\
\times P^{k+1}(K) \backslash\left\{z^{k+1}\right\} \\
\times P^{k+1}(K) \backslash\left\{\widetilde{P}^{k+1}(x, y)\right\}\end{array}$ & $\overline{P^{k}(K)}$ & $\overline{Y^{k+1}(K)}$ & $\overline{P^{k}(K)}$ & $\overline{P^{k+1}(F) \backslash\left\{z^{k+1}\right\}}$ & $\overline{P^{k}(F)}$ \\
\hline \multicolumn{6}{|c|}{$\mathrm{RT}_{\langle\mathbf{k}\rangle}$} \\
\hline $\boldsymbol{V}(K)$ & $W(K)$ & $\widetilde{\boldsymbol{V}}(K)$ & $\widetilde{W}(K)$ & $M_{V}(F)$ & $M_{H}(F)$ \\
\hline $\begin{array}{r}P^{k+1 \mid k}(K) \\
\times P^{k+1 \mid k}(K) \\
\times P^{k \mid k+1}(K)\end{array}$ & 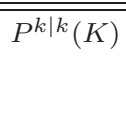 & 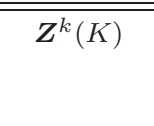 & " & $\overline{P^{k+1, k}(F)}$ & $\overline{P^{k}(F)}$ \\
\hline \multicolumn{6}{|c|}{$\mathrm{HDG}_{\langle\mathbf{k}\rangle}$} \\
\hline $\boldsymbol{V}(K)$ & $W(K)$ & $\tilde{\boldsymbol{V}}(K)$ & $\widetilde{W}(K)$ & $M_{V}(F)$ & $M_{H}(F)$ \\
\hline $\begin{array}{c}\boldsymbol{P}^{k}(K) \\
\oplus \nabla \times\left\{(y,-x, 0) z \widetilde{P}^{k}(K)\right\}\end{array}$ & 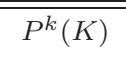 & $\overline{P^{k-1}(K)}$ & $\overline{P^{k-1}(K)}$ & $\overline{P P^{k}(F)}$ & $\overline{P P^{k}(F)}$ \\
\hline
\end{tabular}
convergence. The methods $\mathbf{B D F M} \mathbf{M}_{\langle\mathbf{k}+\mathbf{1}\rangle}$ and $\mathbf{R T}_{\langle\mathbf{k}\rangle}$ were introduced in [14; the last one is a new $\mathbf{H D G}_{\langle k\rangle}$ element.

TABLE 9. Methods for which $K$ is a prism.

TABLE 10. Orders of convergence for methods for which $K$ is a prism.

\begin{tabular}{ccccccc}
\hline method & $\partial K_{V} \partial K_{W}$ & $\tau$ & $\left\|\boldsymbol{q}-\boldsymbol{q}_{h}\right\|_{\mathcal{T}_{h}}\left\|\Pi_{W} u-u_{h}\right\|_{\mathcal{T}_{h}}\left\|u-u_{h}^{\star}\right\|_{\mathcal{T}_{h}}$ \\
\hline \hline $\mathbf{B D F M}_{\langle k+1\rangle}$ & $\partial K$ & - & 0 & $k+1$ & $k+2$ & $k+2$ \\
$\mathbf{R T}_{\langle k\rangle}$ & $\partial K$ & - & 0 & $k+1$ & $k+2$ & $k+2$ \\
$\mathbf{H D G}_{\langle k\rangle}$ & $\partial K$ & $F_{K}$ & $\mathcal{O}(1),>0$ & $k+1$ & $k+2$ & $k+2$ \\
\hline
\end{tabular}


4.5.2. Verification of the conditions on $\boldsymbol{V}^{\perp}(K) \times W^{\perp}(K)$. The methods $\mathbf{B D F M}_{\langle k+1\rangle}, \mathbf{R} \mathbf{T}_{\langle k\rangle}$ were introduced and studied in [14. It is not difficult to verify that they satisfy all the conditions of the template. Here we only consider the $\mathbf{H D G}_{\langle k\rangle}$ method.

We begin by verifying the dimension count (3.6):

$$
\begin{aligned}
\sum_{F \in \mathcal{F}(K)} \operatorname{dim} M(F) & =5 \operatorname{dim} P_{k}(F) \\
\operatorname{dim} \boldsymbol{V}^{\perp}(K) & =\operatorname{dim} \boldsymbol{V}(K)-\operatorname{dim} \widetilde{\boldsymbol{V}}(K) \\
& \left.=\left(3 \operatorname{dim} P_{k}(K)+\operatorname{dim} \widetilde{P}_{k}(K)\right)-3 \operatorname{dim} P_{k-1}(K)\right) \\
& =4 \operatorname{dim} P_{k}(F), \\
\operatorname{dim} W^{\perp}(K) & =\operatorname{dim} W(K)-\operatorname{dim} \widetilde{W}(K) \\
& =\operatorname{dim} P_{k}(K)-\operatorname{dim} P_{k-1}(K) \\
& =\operatorname{dim} P_{k}(F),
\end{aligned}
$$

and the condition (3.6) follows.

The property (4.1b) holds since the spaces $W(K)$ and $W^{\perp}(K)$ are the same as those for simplexes. It remains to verify (4.1a). Assume that the two horizontal faces of the prism $K$ lie on the planes $z=a$ and $z=b$, respectively. By the definition of $\boldsymbol{V}(K)$, for any $\boldsymbol{v} \in \boldsymbol{V}^{\perp}(K)$, we can write

$$
\boldsymbol{v}=\left(p_{1}+x \frac{\partial z \tilde{p}}{\partial z}, p_{2}+y \frac{\partial z \tilde{p}}{\partial z}, p_{3}-z\left(\frac{\partial x \widetilde{p}}{\partial x}+\frac{\partial y \widetilde{p}}{\partial y}\right)\right),
$$

where $\left(p_{1}, p_{2}, p_{3}\right) \in \boldsymbol{P}^{k}(K)$ and that $\widetilde{p} \in \widetilde{P}^{k}(K)$. Noting that $v_{3} \in P^{k+1}(K)$ and $\left.\boldsymbol{v} \cdot \boldsymbol{n}\right|_{z=a, b}=\left.v_{3}\right|_{z=a, b}=0$, we can apply the same argument used in the case of cubic elements to get that $v_{3}=0$ in $K$. This means that

$$
p_{3}=0, \quad \frac{\partial x \widetilde{p}}{\partial x}+\frac{\partial y \tilde{p}}{\partial y}=0 .
$$

Since $\widetilde{p}=\sum_{l+m+n=k} a_{l m n} x^{l} y^{m} z^{n}$, we have

$$
\frac{\partial x \widetilde{p}}{\partial x}+\frac{\partial y \widetilde{p}}{\partial y}=\sum_{l+m+n=k}(l+m+2) a_{l m n} x^{l} y^{m} z^{n}
$$

and so, all the coefficients $a_{l m n}=0$. We then we have $\widetilde{p}=0$. Therefore, $v_{1}, v_{2} \in$ $P^{k}(K)$. It is now very easy to conclude that $v_{1}=v_{2}=0$ by using the fact that $\boldsymbol{v} \cdot \boldsymbol{n}=0$ on the vertical faces and $\boldsymbol{v} \in \boldsymbol{V}^{\perp}(K)$. This shows that property 4.1a) also holds.

4.6. A remark on the construction of the superconvergent methods. Let us briefly discuss the use of the template proposed for the construction of the superconvergent methods. In the simplex case, we have

$$
\begin{aligned}
& W(K)=P^{k}(K), \boldsymbol{V}=\boldsymbol{P}^{k}(K), M(F)=P^{k}(F), \\
& \widetilde{W}(K)=P^{k-1}(K), \widetilde{\boldsymbol{V}}(K)=\boldsymbol{P}^{k-1}(K),
\end{aligned}
$$

and

$$
\sum_{F \in \mathcal{F}(F)} \operatorname{dim} M(F)=\left(\operatorname{dim} W^{\perp}(K)+\operatorname{dim} \boldsymbol{V}^{\perp}(K)\right) .
$$


If we were to keep the same local spaces for, say, rectangular elements, the condition (3.6) would not be satisfied because we have one additional face. Indeed, we would have

$$
\sum_{F \in \mathcal{F}(F)} \operatorname{dim} M(F)=\left(\operatorname{dim} W^{\perp}(K)+\operatorname{dim} \boldsymbol{V}^{\perp}(K)\right)+\operatorname{dim} P^{k}(F) .
$$

As discussed in Subsection 3.1, to remedy this situation, we must modify the local space $\boldsymbol{V}(K)$ by adding new basis functions (i) which are divergence-free, (ii) which are such that their normal component on the face $F$ lies on $M(F)=P^{k}(F)$, and (iii) whose behavior in the element is controlled by the behavior on the normal component on its boundary. The condition (i), suggests to add $\operatorname{dim} P^{k}(F)=k+1$, new basis functions of the form

$$
\nabla \times f=\left(-\frac{\partial f}{\partial y}, \frac{\partial f}{\partial x}\right)
$$

where $f \in \widetilde{P}^{k+2}(K)$. The conditions (ii) and (iii) now suggest to take $f$ in the space $x y \widetilde{P}^{k}(K)$. All the new superconvergent elements were found in a similar manner.

\section{Proofs of the estimates of the projection of the ERrors}

In this section we provide detailed proofs for our a priori error estimates. The main idea is to work with the following projection of the errors:

$$
\begin{aligned}
\boldsymbol{e}_{q} & :=\boldsymbol{\Pi}_{V} \boldsymbol{q}-\boldsymbol{q}_{h}, \\
e_{u} & :=\Pi_{W} u-u_{h}, \\
\boldsymbol{e}_{\widehat{q}} \cdot \boldsymbol{n} & :=P_{M}\left(\boldsymbol{q} \cdot \boldsymbol{n}-\widehat{\boldsymbol{q}}_{h} \cdot \boldsymbol{n}\right), \\
e_{\widehat{u}} & :=P_{M} u-\widehat{u}_{h} .
\end{aligned}
$$

Here, $P_{M}$ is the $L^{2}$-projection from $L^{2}\left(\varepsilon_{h}\right)$ into $M_{h}$. We abuse the notation for the sake of simplicity and denote with the same symbol the $L^{2}$-projection from $L^{2}\left(\partial \mathcal{T}_{h}\right)$ into the space

$$
\left\{w \in L^{2}\left(\partial \mathcal{T}_{h}\right):\left.\left(\left.w\right|_{\partial K}\right)\right|_{F} \in M(F) \text { for all faces } F \text { of } K \text { and all } K \in \mathcal{T}_{h}\right\} .
$$

We begin by obtaining the equations satisfied by these projections. We then use an energy argument to obtain an estimate of $\boldsymbol{e}_{q}$; this would prove Theorem 2.1. To obtain an estimate of $e_{u}$ and prove Theorem 2.2. we employ an elliptic duality. Finally, we obtain the estimate of $u-u_{h}^{*}$ of Theorem 2.3 by using a simple element-by-element argument.

Step 1: The equations for the projection of the errors. We begin our error analysis with the following auxiliary result.

Lemma 5.1. Suppose that the orthogonality properties of the projection $\Pi_{h}$ and the properties of the traces of the local spaces of Assumption A are satisfied. Then, we have

$$
\begin{aligned}
\left(\boldsymbol{e}_{q}, \boldsymbol{v}\right)_{\mathcal{T}_{h}}-\left(e_{u}, \nabla \cdot \boldsymbol{v}\right)_{\mathcal{T}_{h}}+\left\langle e_{\widehat{u}}, \boldsymbol{v} \cdot \boldsymbol{n}\right\rangle_{\partial \mathcal{T}_{h}} & =\left(\boldsymbol{\Pi}_{V} \boldsymbol{q}-\boldsymbol{q}, \boldsymbol{v}\right)_{\mathcal{T}_{h}} \\
-\left(\boldsymbol{e}_{q}, \nabla w\right)_{\mathcal{T}_{h}}+\left\langle\boldsymbol{e}_{\widehat{q}} \cdot \boldsymbol{n}, w\right\rangle_{\partial \mathcal{T}_{h}} & =0 \\
\left\langle\boldsymbol{e}_{\widehat{q}} \cdot \boldsymbol{n}, \mu\right\rangle_{\partial \mathcal{T}_{h} \backslash \partial \Omega} & =0 \\
\left\langle e_{\widehat{u}}, \mu\right\rangle_{\partial \Omega} & =0
\end{aligned}
$$


for all $(\boldsymbol{v}, w, \mu) \in \boldsymbol{V}_{h} \times W_{h} \times M_{h}$. Moreover,

$$
\boldsymbol{e}_{\widehat{q}} \cdot \boldsymbol{n}=\boldsymbol{e}_{q} \cdot \boldsymbol{n}+P_{M}\left(\alpha\left(e_{u}-e_{\widehat{u}}\right)\right) \quad \text { on } \quad \partial \mathcal{T}_{h} .
$$

Proof. Let us begin by noting that the exact solution $(\boldsymbol{q}, u)$ satisfies the equations

$$
\begin{aligned}
&(\boldsymbol{q}, \boldsymbol{v})_{\mathcal{T}_{h}-(u, \nabla \cdot \boldsymbol{v})_{\mathcal{T}_{h}}+\langle u, \boldsymbol{v} \cdot \boldsymbol{n}\rangle_{\partial \mathcal{T}_{h}}}=0, \\
&-(\boldsymbol{q}, \nabla w)_{\mathcal{T}_{h}}+\langle\boldsymbol{q} \cdot \boldsymbol{n}, w\rangle_{\partial \mathcal{T}_{h}}=(f, w)_{\mathcal{T}_{h}}, \\
&\langle\boldsymbol{q} \cdot \boldsymbol{n}, \mu\rangle_{\partial \mathcal{T}_{h} \backslash \partial \Omega}=0, \\
&\langle u, \mu\rangle_{\partial \Omega}=\langle g, \mu\rangle_{\partial \Omega},
\end{aligned}
$$

for all $(\boldsymbol{v}, w, \mu) \in \boldsymbol{V}_{h} \times W_{h} \times M_{h}$. By the orthogonality properties $(A .1)$ and (A.2) of the projection $\Pi_{h}=\left(\boldsymbol{\Pi}_{V}, \Pi_{W}\right)$, we obtain that

$$
\begin{aligned}
(\boldsymbol{q}, \boldsymbol{v})_{\mathcal{T}_{h}}-\left(\Pi_{W} u, \nabla \cdot \boldsymbol{v}\right)_{\mathcal{T}_{h}}+\langle u, \boldsymbol{v} \cdot \boldsymbol{n}\rangle_{\partial \mathcal{T}_{h}} & =0 \\
-\left(\boldsymbol{\Pi}_{V} \boldsymbol{q}, \nabla w\right)_{\mathcal{T}_{h}}+\langle\boldsymbol{q} \cdot \boldsymbol{n}, w\rangle_{\partial \mathcal{T}_{h}} & =(f, w)_{\mathcal{T}_{h}}, \\
\langle\boldsymbol{q} \cdot \boldsymbol{n}, \mu\rangle_{\partial \mathcal{T}_{h} \backslash \partial \Omega} & =0 \\
\langle u, \mu\rangle_{\partial \Omega} & =\langle g, \mu\rangle_{\partial \Omega},
\end{aligned}
$$

for all $(\boldsymbol{v}, w, \mu) \in \boldsymbol{V}_{h} \times W_{h} \times M_{h}$. Moreover, since $P_{M}$ is the $L^{2}$-projection into $M_{h}$, we get, by the properties $(A .4)$ and $(A .5)$ of the traces of the local spaces, that

$$
\begin{aligned}
(\boldsymbol{q}, \boldsymbol{v})_{\mathcal{T}_{h}}-\left(\Pi_{W} u, \nabla \cdot \boldsymbol{v}\right)_{\mathcal{T}_{h}}+\left\langle P_{M} u, \boldsymbol{v} \cdot \boldsymbol{n}\right\rangle_{\partial \mathcal{T}_{h}} & =0 \\
-\left(\boldsymbol{\Pi}_{V} \boldsymbol{q}, \nabla w\right)_{\mathcal{T}_{h}}+\left\langle P_{M}(\boldsymbol{q} \cdot \boldsymbol{n}), w\right\rangle_{\partial \mathcal{T}_{h}} & =(f, w)_{\mathcal{T}_{h}}, \\
\left\langle P_{M}(\boldsymbol{q} \cdot \boldsymbol{n}), \mu\right\rangle_{\partial \mathcal{T}_{h} \backslash \partial \Omega} & =0 \\
\left\langle P_{M} u, \mu\right\rangle_{\partial \Omega} & =\langle g, \mu\rangle_{\partial \Omega},
\end{aligned}
$$

for all $(\boldsymbol{v}, w, \mu) \in \boldsymbol{V}_{h} \times W_{h} \times M_{h}$. Subtracting the first four equations defining the weak formulation of the HDG method (1.2) from the above equations, respectively, we obtain the equations for the projection of the errors.

It remains to prove the identity for $\boldsymbol{e}_{\widehat{q}}$. We have

$$
\begin{aligned}
\boldsymbol{e}_{\widehat{q}} \cdot \boldsymbol{n} & =P_{M}(\boldsymbol{q} \cdot \boldsymbol{n})-P_{M}\left(\widehat{\boldsymbol{q}}_{h} \cdot \boldsymbol{n}\right) \\
& =P_{M}\left(\boldsymbol{\Pi}_{V} \boldsymbol{q} \cdot \boldsymbol{n}+\alpha\left(\Pi_{W} u-P_{M} u\right)\right)-P_{M}\left(\widehat{\boldsymbol{q}}_{h} \cdot \boldsymbol{n}\right),
\end{aligned}
$$

by the orthogonality property $(A .3)$ of the projection $\Pi_{h}$. Inserting the definition of the numerical trace $\widehat{\boldsymbol{q}}_{h} \cdot \boldsymbol{n},(1.3)$, we get

$$
\begin{aligned}
\boldsymbol{e}_{\widehat{q}} \cdot \boldsymbol{n} & =P_{M}\left(\boldsymbol{e}_{q} \cdot \boldsymbol{n}+\alpha\left(e_{u}-e_{\widehat{u}}\right)\right) \\
& =\boldsymbol{e}_{q} \cdot \boldsymbol{n}+P_{M}\left(\alpha\left(e_{u}-e_{\widehat{u}}\right)\right),
\end{aligned}
$$

by the property $(A .4)$ of the trace of the local spaces. This completes the proof.

Step 2: The energy argument for $\boldsymbol{e}_{q}$. We are now ready to obtain the upper bound of the $L^{2}$-norm of $\boldsymbol{e}_{q}$. We proceed as follows. Taking $\boldsymbol{v}:=\boldsymbol{e}_{q}$ in the error equation (5.1a), $w:=e_{u}$ in the error equation (5.1b), $\mu:=-e_{\widehat{u}}$ in the error equation (5.1c), and $\mu:=-P_{M}\left(\boldsymbol{e}_{\widehat{q}} \cdot \boldsymbol{n}\right)$ in the error equation (5.1d), and adding the resulting equations up, we obtain

$$
\left(\boldsymbol{e}_{q}, \boldsymbol{e}_{q}\right)_{\mathcal{T}_{h}}+\Theta_{h}=\left(\boldsymbol{\Pi}_{V} \boldsymbol{q}-\boldsymbol{q}, \boldsymbol{e}_{q}\right)_{\mathcal{T}_{h}},
$$


where

$$
\begin{aligned}
\Theta_{h}:= & \left\langle e_{\widehat{u}}, \boldsymbol{e}_{q} \cdot \boldsymbol{n}\right\rangle_{\partial \mathcal{T}_{h}}+\left\langle\boldsymbol{e}_{\widehat{q}} \cdot \boldsymbol{n}, e_{u}\right\rangle_{\partial \mathcal{T}_{h}} \\
& -\left\langle\boldsymbol{e}_{q} \cdot \boldsymbol{n}, e_{u}\right\rangle_{\partial \mathcal{T}_{h}}-\left\langle\boldsymbol{e}_{\widehat{q}} \cdot \boldsymbol{n}, e_{\widehat{u}}\right\rangle_{\partial \mathcal{T}_{h} \backslash \partial \Omega}-\left\langle P_{M}\left(\boldsymbol{e}_{\widehat{q}} \cdot \boldsymbol{n}\right), e_{\widehat{u}}\right\rangle_{\partial \Omega} .
\end{aligned}
$$

By the definition of the projection $P_{M}$, we get that

$$
\begin{aligned}
\Theta_{h}= & \left\langle e_{\widehat{u}}, \boldsymbol{e}_{q} \cdot \boldsymbol{n}\right\rangle_{\partial \mathcal{T}_{h}}+\left\langle\boldsymbol{e}_{\widehat{q}} \cdot \boldsymbol{n}, e_{u}\right\rangle_{\partial \mathcal{T}_{h}} \\
& -\left\langle\boldsymbol{e}_{q} \cdot \boldsymbol{n}, e_{u}\right\rangle_{\partial \mathcal{T}_{h}}-\left\langle\boldsymbol{e}_{\widehat{q}} \cdot \boldsymbol{n}, e_{\widehat{u}}\right\rangle_{\partial \mathcal{T}_{h} \backslash \partial \Omega}-\left\langle\boldsymbol{e}_{\widehat{q}} \cdot \boldsymbol{n}, e_{\widehat{u}}\right\rangle_{\partial \Omega} \\
= & \left\langle\left(\boldsymbol{e}_{\widehat{q}}-\boldsymbol{e}_{q}\right) \cdot \boldsymbol{n}, e_{u}-e_{\widehat{u}}\right\rangle_{\partial \mathcal{T}_{h}} \\
= & \left\langle P_{M}\left(\alpha\left(e_{u}-e_{\widehat{u}}\right)\right), e_{u}-e_{\widehat{u}}\right\rangle_{\partial \mathcal{T}_{h}},
\end{aligned}
$$

by the identity (5.2) of Lemma 5.1. Finally, by the definition of the projection $P_{M}$ and the property $(A .5)$ of the traces of the local spaces, we obtain that

$$
\Theta_{h}=\left\langle\alpha\left(e_{u}-e_{\widehat{u}}\right), e_{u}-e_{\widehat{u}}\right\rangle_{\partial \mathcal{T}_{h}}
$$

Since $\Theta_{h} \geq 0$, by the semi-positivity property (A.6) of the local stabilization operator $\alpha$, we have that

$$
\begin{aligned}
\left\|\boldsymbol{e}_{q}\right\|_{\mathcal{T}_{h}}^{2} & \leq\left(\boldsymbol{\Pi}_{V} \boldsymbol{q}-\boldsymbol{q}, \boldsymbol{e}_{q}\right)_{\mathcal{T}_{h}} \\
& \leq\left\|\boldsymbol{\Pi}_{V} \boldsymbol{q}-\boldsymbol{q}\right\|_{\mathcal{T}_{h}}\left\|\boldsymbol{e}_{q}\right\|_{\mathcal{T}_{h}},
\end{aligned}
$$

and the result follows. This completes the proof of Theorem 2.1

Step 3: The elliptic duality argument for $e_{u}$. The estimate of $e_{u}$ will follow from the following identity.

Lemma 5.2. Suppose that the assumptions of Lemma 5.1 are satisfied. Then, we have

$$
\left(e_{u}, \eta\right)_{\mathcal{T}_{h}}=\left(\boldsymbol{q}-\boldsymbol{\Pi}_{V} \boldsymbol{q}, \boldsymbol{\Pi}_{V}^{*} \boldsymbol{\theta}\right)_{\mathcal{T}_{h}}-\left(\boldsymbol{e}_{q}, \boldsymbol{\theta}-\boldsymbol{\Pi}_{V}^{*} \boldsymbol{\theta}\right)_{\mathcal{T}_{h}},
$$

where $(\phi, \boldsymbol{\theta})$ is the solution of dual problem (2.2).

Proof. We begin by using the second equation (2.2b) of the dual problem to write that

$$
\begin{aligned}
\left(e_{u}, \eta\right)_{\mathcal{T}_{h}} & =\left(e_{u}, \nabla \cdot \boldsymbol{\theta}\right)_{\mathcal{T}_{h}} \\
& =\left(e_{u}, \nabla \cdot \boldsymbol{\theta}\right)_{\mathcal{T}_{h}}-\left(\boldsymbol{e}_{q}, \boldsymbol{\theta}\right)_{\mathcal{T}_{h}}-\left(\boldsymbol{e}_{q}, \nabla \phi\right)_{\mathcal{T}_{h}}
\end{aligned}
$$

by the first equation (2.2a) of the dual problem. This implies that

$$
\begin{aligned}
\left(e_{u}, \eta\right)_{\mathcal{T}_{h}}= & \left(e_{u}, \nabla \cdot \boldsymbol{\Pi}_{V}^{*} \boldsymbol{\theta}\right)_{\mathcal{T}_{h}}-\left(\boldsymbol{e}_{q}, \boldsymbol{\Pi}_{V}^{*} \boldsymbol{\theta}\right)_{\mathcal{T}_{h}}-\left(\boldsymbol{e}_{q}, \nabla \Pi_{W}^{*} \phi\right)_{\mathcal{T}_{h}} \\
& +\left(e_{u}, \nabla \cdot\left(\boldsymbol{\theta}-\boldsymbol{\Pi}_{V}^{*} \boldsymbol{\theta}\right)\right)_{\mathcal{T}_{h}}-\left(\boldsymbol{e}_{q}, \boldsymbol{\theta}-\boldsymbol{\Pi}_{V}^{*} \boldsymbol{\theta}\right)_{\mathcal{T}_{h}}-\left(\boldsymbol{e}_{q}, \nabla\left(\phi-\Pi_{W}^{*} \phi\right)\right)_{\mathcal{T}_{h}} .
\end{aligned}
$$

Taking $\boldsymbol{v}:=\boldsymbol{\Pi}_{V}^{*} \boldsymbol{\theta}$ in the first error equation, (5.1a), and $w:=\Pi_{W}^{*} \phi$ in the second, (5.1b), we obtain that

$$
\begin{aligned}
\left(e_{u}, \eta\right)_{\mathcal{T}_{h}}= & \left(\boldsymbol{q}-\boldsymbol{\Pi}_{V} \boldsymbol{q}, \boldsymbol{\Pi}_{V}^{*} \boldsymbol{\theta}\right)_{\mathcal{T}_{h}}+\left\langle e_{\widehat{u}}, \boldsymbol{\Pi}_{V}^{*} \boldsymbol{\theta} \cdot \boldsymbol{n}\right\rangle_{\partial \mathcal{T}_{h}}-\left\langle\boldsymbol{e}_{\widehat{q}} \cdot \boldsymbol{n}, \Pi_{W}^{*} \phi\right\rangle_{\partial \mathcal{T}_{h}} \\
& +\left(e_{u}, \nabla \cdot\left(\boldsymbol{\theta}-\boldsymbol{\Pi}_{V}^{*} \boldsymbol{\theta}\right)\right)_{\mathcal{T}_{h}}-\left(\boldsymbol{e}_{q}, \boldsymbol{\theta}-\boldsymbol{\Pi}_{V}^{*} \boldsymbol{\theta}\right)_{\mathcal{T}_{h}}-\left(\boldsymbol{e}_{q}, \nabla\left(\phi-\Pi_{W}^{*} \phi\right)\right)_{\mathcal{T}_{h}},
\end{aligned}
$$

and, after simple algebraic manipulations, that

$$
\left(e_{u}, \eta\right)_{\mathcal{T}_{h}}=\left(\boldsymbol{q}-\boldsymbol{\Pi}_{V} \boldsymbol{q}, \boldsymbol{\Pi}_{V}^{*} \boldsymbol{\theta}\right)_{\mathcal{T}_{h}}-\left(\boldsymbol{e}_{q}, \boldsymbol{\theta}-\boldsymbol{\Pi}_{V}^{*} \boldsymbol{\theta}\right)_{\mathcal{T}_{h}}+\mathbb{T},
$$


where

$$
\begin{aligned}
\mathbb{T}:= & \left\langle e_{\widehat{u}}, \boldsymbol{\Pi}_{V}^{*} \boldsymbol{\theta} \cdot \boldsymbol{n}\right\rangle_{\partial \mathcal{T}_{h}}-\left\langle\boldsymbol{e}_{\widehat{q}} \cdot \boldsymbol{n}, \Pi_{W}^{*} \phi\right\rangle_{\partial \mathcal{T}_{h}} \\
& +\left(e_{u}, \nabla \cdot\left(\boldsymbol{\theta}-\boldsymbol{\Pi}_{V}^{*} \boldsymbol{\theta}\right)\right)_{\mathcal{T}_{h}}-\left(\boldsymbol{e}_{q}, \nabla\left(\phi-\Pi_{W}^{*} \phi\right)\right)_{\mathcal{T}_{h}} .
\end{aligned}
$$

It remains to prove that $\mathbb{T}=0$.

To do that, we integrate by parts and use the orthogonality properties $(A .1)$ and (A.2) of the projection $\Pi_{h}^{*}$ to get

$$
\begin{aligned}
\mathbb{T}= & \left\langle e_{\widehat{u}}, \boldsymbol{\Pi}_{V}^{*} \boldsymbol{\theta} \cdot \boldsymbol{n}\right\rangle_{\partial \mathcal{T}_{h}}-\left\langle\boldsymbol{e}_{\widehat{q}} \cdot \boldsymbol{n}, \Pi_{W}^{*} \phi\right\rangle_{\partial \mathcal{T}_{h}} \\
& +\left\langle e_{u},\left(\boldsymbol{\theta}-\Pi_{V}^{*} \boldsymbol{\theta}\right) \cdot \boldsymbol{n}\right\rangle_{\partial \mathcal{T}_{h}}-\left\langle\boldsymbol{e}_{q} \cdot \boldsymbol{n},\left(\phi-\Pi_{W}^{*} \phi\right)\right\rangle_{\partial \mathcal{T}_{h}} \\
= & \left\langle e_{\widehat{u}}-e_{u},\left(\boldsymbol{\Pi}_{V}^{*} \boldsymbol{\theta}-\boldsymbol{\theta}\right) \cdot \boldsymbol{n}\right\rangle_{\partial \mathcal{T}_{h}}-\left\langle\left(\boldsymbol{e}_{\widehat{q}}-\boldsymbol{e}_{q}\right) \cdot \boldsymbol{n}, \Pi_{W}^{*} \phi-\phi\right\rangle_{\partial \mathcal{T}_{h}} \\
& +\left\langle e_{\widehat{u}}, \boldsymbol{\theta} \cdot \boldsymbol{n}\right\rangle_{\partial \mathcal{T}_{h}}-\left\langle\boldsymbol{e}_{\widehat{q}} \cdot \boldsymbol{n}, \phi\right\rangle_{\partial \mathcal{T}_{h}} \\
= & \left\langle e_{\widehat{u}}-e_{u},\left(\boldsymbol{\Pi}_{V}^{*} \boldsymbol{\theta}-\boldsymbol{\theta}\right) \cdot \boldsymbol{n}\right\rangle_{\partial \mathcal{T}_{h}}-\left\langle\left(\boldsymbol{e}_{\widehat{q}}-\boldsymbol{e}_{q}\right) \cdot \boldsymbol{n}, \Pi_{W}^{*} \phi-\phi\right\rangle_{\partial \mathcal{T}_{h}} .
\end{aligned}
$$

Indeed, the fact that $\left\langle e_{\widehat{u}}, \boldsymbol{\theta} \cdot \boldsymbol{n}\right\rangle_{\partial \mathcal{T}_{h}}=0$ is proven as follows. Since $e_{\widehat{u}}$ is single valued on $\mathcal{E}_{h}$ and $\boldsymbol{\theta}$ lies in $\boldsymbol{H}$ (div), we have that

$$
\begin{aligned}
\left\langle e_{\widehat{u}}, \boldsymbol{\theta} \cdot \boldsymbol{n}\right\rangle_{\partial \mathcal{T}_{h}} & =\left\langle e_{\widehat{u}}, \boldsymbol{\theta} \cdot \boldsymbol{n}\right\rangle_{\partial \Omega} \\
& =\left\langle e_{\widehat{u}}, P_{M}(\boldsymbol{\theta} \cdot \boldsymbol{n})\right\rangle_{\partial \Omega},
\end{aligned}
$$

by the definition of the projection $P_{M}$. The above quantity is equal to zero by the fourth error equation (5.1d) with $\mu:=P_{M}(\boldsymbol{\theta} \cdot \boldsymbol{n})$.

We can prove that $\left\langle\boldsymbol{e}_{\widehat{q}} \cdot \boldsymbol{n}, \phi\right\rangle_{\partial \mathcal{T}_{h}}=0$ as follows. By the definition of the projection $P_{M}$

$$
\begin{aligned}
\left\langle\boldsymbol{e}_{\widehat{q}} \cdot \boldsymbol{n}, \phi\right\rangle_{\partial \mathcal{T}_{h}} & =\left\langle\boldsymbol{e}_{\widehat{q}} \cdot \boldsymbol{n}, P_{M}(\phi)\right\rangle_{\partial \mathcal{T}_{h}} \\
& =\left\langle\boldsymbol{e}_{\widehat{q}} \cdot \boldsymbol{n}, \phi\right\rangle_{\partial \Omega},
\end{aligned}
$$

by the third error equation (5.1c) with $\mu:=P_{M}(\phi)$. Finally, by the third equation (2.2c) of the dual problem, we have that $\phi=0$ on $\partial \Omega$ and the result follows.

Now, inserting the expression for $\boldsymbol{e}_{\widehat{q}},(\underline{5.2})$, given by Lemma 5.1 in the last expression for $\mathbb{T}$, we get

$$
\begin{aligned}
\mathbb{T} & =\left\langle e_{\widehat{u}}-e_{u},\left(\Pi_{V}^{*} \boldsymbol{\theta}-\boldsymbol{\theta}\right) \cdot \boldsymbol{n}\right\rangle_{\partial \mathcal{T}_{h}}-\left\langle P_{M}\left(\alpha\left(e_{u}-e_{\widehat{u}}\right)\right), \Pi_{W}^{*} \phi-\phi\right\rangle_{\partial \mathcal{T}_{h}} \\
& =\left\langle e_{\widehat{u}}-e_{u},\left(\boldsymbol{\Pi}_{V}^{*} \boldsymbol{\theta}-\boldsymbol{\theta}\right) \cdot \boldsymbol{n}\right\rangle_{\partial \mathcal{T}_{h}}-\left\langle\alpha\left(e_{u}-e_{\widehat{u}}\right), \Pi_{W}^{*} \phi-P_{M} \phi\right\rangle_{\partial \mathcal{T}_{h}},
\end{aligned}
$$

by the definition of the projection $P_{M}$ and the second property $(A .5)$ of the traces of the local spaces. Then

$$
\begin{aligned}
\mathbb{T} & =\left\langle e_{\widehat{u}}-e_{u},\left(\Pi_{V}^{*} \boldsymbol{\theta}-\boldsymbol{\theta}\right) \cdot \boldsymbol{n}\right\rangle_{\partial \mathcal{T}_{h}}-\left\langle e_{u}-e_{\widehat{u}}, \alpha^{*}\left(\Pi_{W}^{*} \phi-P_{M} \phi\right)\right\rangle_{\partial \mathcal{T}_{h}} \\
& =\left\langle e_{\widehat{u}}-e_{u},\left(\boldsymbol{\Pi}_{V}^{*} \boldsymbol{\theta}-\boldsymbol{\theta}\right) \cdot \boldsymbol{n}+\alpha^{*}\left(\Pi_{W}^{*} \phi-P_{M} \phi\right)\right\rangle_{\partial \mathcal{T}_{h}} \\
& =0,
\end{aligned}
$$

by the property $(A .5)$ of the traces of the local spaces and the orthogonality property (A.3) of the projection $\Pi_{h}^{*}$. This completes the proof.

Step 4: The estimate for $e_{u}$. We are now ready to obtain the estimate of the $L^{2}$-norm of $e_{u}$ and prove Theorem 2.2 ,

We start by taking $\eta=e_{u}$ in the identity of Lemma 5.2 to obtain

$$
\begin{aligned}
\left\|e_{u}\right\|_{\mathcal{T}_{h}}^{2} & =\left(\boldsymbol{q}-\boldsymbol{\Pi}_{V} \boldsymbol{q}, \boldsymbol{\Pi}_{V}^{*} \boldsymbol{\theta}\right)_{\mathcal{T}_{h}}-\left(\boldsymbol{e}_{q}, \boldsymbol{\theta}-\boldsymbol{\Pi}_{V}^{*} \boldsymbol{\theta}\right)_{\mathcal{T}_{h}} \\
& =\left(\boldsymbol{q}-\boldsymbol{\Pi}_{V} \boldsymbol{q}, \boldsymbol{\theta}\right)_{\mathcal{T}_{h}}-\left(\boldsymbol{q}-\boldsymbol{q}_{h}, \boldsymbol{\theta}-\boldsymbol{\Pi}_{V}^{*} \boldsymbol{\theta}\right)_{\mathcal{T}_{h}} .
\end{aligned}
$$


If we now use the orthogonality property $(A .1)$ of the projection $\Pi_{h}$ and the property $(B .2)$ that the space $W(K)$ is not too small, we get

$$
\left\|e_{u}\right\|_{\mathcal{T}_{h}}^{2}=\left(\boldsymbol{q}-\boldsymbol{\Pi}_{V} \boldsymbol{q}, \boldsymbol{\theta}-\boldsymbol{P}_{0} \boldsymbol{\theta}\right)_{\mathcal{T}_{h}}-\left(\boldsymbol{q}-\boldsymbol{q}_{h}, \boldsymbol{\theta}-\boldsymbol{\Pi}_{V}^{*} \boldsymbol{\theta}\right)_{\mathcal{T}_{h}},
$$

where $\boldsymbol{P}_{0}$ is the $L^{2}$-projection into $\left\{\boldsymbol{v} \in \boldsymbol{L}^{2}(\Omega):\left.\boldsymbol{v}\right|_{K} \in \boldsymbol{P}^{0}(K) \forall K \in \mathcal{T}_{h}\right\}$. By the Cauchy-Schwartz inequality, we get

$$
\begin{aligned}
\left\|e_{u}\right\|_{\mathcal{T}_{h}}^{2} & \leq\left\|\boldsymbol{q}-\boldsymbol{\Pi}_{V} \boldsymbol{q}\right\|_{\mathcal{T}_{h}}\left\|\boldsymbol{\theta}-\boldsymbol{P}_{0} \boldsymbol{\theta}\right\|_{\mathcal{T}_{h}}+\left\|\boldsymbol{q}-\boldsymbol{q}_{h}\right\|_{\mathcal{T}_{h}}\left\|\boldsymbol{\theta}-\boldsymbol{\Pi}_{V}^{*} \boldsymbol{\theta}\right\|_{\mathcal{T}_{h}} \\
& \leq\left(\left\|\boldsymbol{\theta}-\boldsymbol{P}_{0} \boldsymbol{\theta}\right\|_{\mathcal{T}_{h}}+2\left\|\boldsymbol{\theta}-\boldsymbol{\Pi}_{V}^{*} \boldsymbol{\theta}\right\|_{\mathcal{T}_{h}}\right)\left\|\boldsymbol{q}-\boldsymbol{\Pi}_{V} \boldsymbol{q}\right\|_{\mathcal{T}_{h}} \\
& \leq C h\left(|\boldsymbol{\theta}|_{1, \mathcal{T}_{h}}+|\phi|_{2, \mathcal{T}_{h}}\right)\left\|\boldsymbol{q}-\boldsymbol{\Pi}_{V} \boldsymbol{q}\right\|_{\mathcal{T}_{h}}
\end{aligned}
$$

by the standard approximation properties of the projection $\boldsymbol{P}_{0}$ and by the approximation property (B.1) of the dual projection $\Pi_{h}^{*}$. Finally, by the elliptic regularity property (2.1) with $\eta:=e_{u}$, we conclude that

$$
\left\|e_{u}\right\|_{\mathcal{T}_{h}}^{2} \leq C C_{\text {app }}^{*} h\left\|e_{u}\right\|_{\mathcal{T}_{h}}\left\|\boldsymbol{q}-\boldsymbol{\Pi}_{V} \boldsymbol{q}\right\|_{\mathcal{T}_{h}},
$$

and the estimate follows. This completes the proof of Theorem 2.2

Step 5: The estimate for $u-u^{*}$. By the Poincaré-Friedrichs inequality, we have that

$$
\left\|u-u_{h}^{*}\right\|_{K} \leq\left\|\overline{u-u_{h}^{*}}\right\|_{K}+C h_{K}\left\|\nabla\left(u-u_{h}^{*}\right)\right\|_{K},
$$

where $\bar{w}$ is the average of $w$ over $K$. But $\overline{u_{h}^{*}}=\overline{u_{h}}$, by the second equation defining $u_{h}^{*}$, (1.4c), and $\bar{u}=\overline{\Pi_{W} u}$ by Assumptions (A.2) and (C.1). This implies that

$$
\left\|u-u_{h}^{*}\right\|_{K} \leq\left\|\Pi_{W} u-u_{h}\right\|_{K}+C h_{K}\left\|\nabla\left(u-u_{h}^{*}\right)\right\|_{K} .
$$

Now, for any $\omega \in \mathcal{W}(K)$, we have that

$$
\begin{aligned}
\left\|\nabla\left(u-u_{h}^{*}\right)\right\|_{K}^{2} & =\left(\nabla\left(u-u_{h}^{*}\right), \nabla(u-\omega)\right)_{K}+\left(\nabla\left(u-u_{h}^{*}\right), \nabla\left(\omega-u_{h}^{*}\right)\right)_{K} \\
& =\left(\nabla\left(u-u_{h}^{*}\right), \nabla(u-\omega)\right)_{K}-\left(\boldsymbol{q}-\boldsymbol{q}_{h}, \nabla\left(\omega-u_{h}^{*}\right)\right)_{K},
\end{aligned}
$$

by the first equation defining the postprocessing $u_{h}^{*}$, (1.4b). Applying the CauchySchwarz inequality, we obtain that

$$
\begin{aligned}
& \left\|\nabla\left(u-u_{h}^{*}\right)\right\|_{K}^{2} \leq\left\|\nabla\left(u-u_{h}^{*}\right)\right\|_{K}\|\nabla(u-\omega)\|_{K}+\left\|\boldsymbol{q}-\boldsymbol{q}_{h}\right\|_{K}\left\|\nabla\left(\omega-u_{h}^{*}\right)\right\|_{K} \\
& \quad \leq\left\|\nabla\left(u-u_{h}^{*}\right)\right\|_{K}\left(\|\nabla(u-\omega)\|_{K}+\left\|\boldsymbol{q}-\boldsymbol{q}_{h}\right\|_{K}\right)+\left\|\boldsymbol{q}-\boldsymbol{q}_{h}\right\|_{K}\|\nabla(\omega-u)\|_{K},
\end{aligned}
$$

and, after simple applications of Young's inequality and some algebraic manipulations, we get that

$$
\left\|\nabla\left(u-u_{h}^{*}\right)\right\|_{K}^{2} \leq 3\left(\left\|\boldsymbol{q}-\boldsymbol{q}_{h}\right\|_{K}^{2}+\|\nabla(u-\omega)\|_{K}^{2}\right) .
$$

This implies that

$$
\left\|u-u_{h}^{*}\right\|_{K} \leq\left\|\Pi_{W} u-u_{h}\right\|_{K}+C h_{K}\left(\left\|\boldsymbol{q}-\boldsymbol{q}_{h}\right\|_{K}+\|\nabla(u-\omega)\|_{K}\right),
$$

and so,

$$
\left\|u-u_{h}^{*}\right\|_{\mathcal{T}_{h}} \leq\left\|\Pi_{W} u-u_{h}\right\|_{\mathcal{T}_{h}}+C h\left(\left\|\boldsymbol{q}-\boldsymbol{q}_{h}\right\|_{\mathcal{T}_{h}}+\|\nabla(u-\omega)\|_{\mathcal{T}_{h}}\right) .
$$

This completes the proof of Theorem 2.3

\section{Concluding Remarks}

We end this paper by discussing some variations on the theoretical results we have proposed in Section 2. 
6.1. Other postprocessings. There are several ways to define a new approximation $u_{h}^{*} \in W^{*}(K)$ for which Theorem 2.3 does hold. The following example is particularly useful when working with the $p$-version of the method; see also [11] and the references therein. On each element $K \in \mathcal{T}_{h}$, the postprocessing $u_{h}^{*}$ is defined as the element of $W^{*}(K)$ such that

$$
\begin{array}{rlrl}
\left(\nabla u_{h}^{*}, \nabla \omega\right)_{K} & =-\left(\boldsymbol{q}_{h}, \nabla \omega\right)_{K} & & \forall \omega \in W^{*}(K):(\omega, \widetilde{w})_{k}=0 \text { for all } \widetilde{w} \in \widetilde{W}(K), \\
\left(u_{h}^{*}, \widetilde{w}\right)_{K}=\left(u_{h}, \widetilde{w}\right)_{K} & \forall \widetilde{w} \in \widetilde{W}(K) .
\end{array}
$$

6.2. Optimal convergence when the local space $W(K)$ is small. When the local space $W(K)$ is small, that is, when it does not satisfy Assumption (B.2), the superconvergence of the projection of the error in the scalar variable, $\Pi_{W} u-u_{h}$, is not guaranteed, and in general it does not take place. Examples of these methods are the $\mathrm{HDG}_{0}$ and the $\mathrm{BDM}_{1}$ methods for simplexes.

However, in this case we can still obtain the optimal order of convergence of $\Pi_{W} u-u_{h}$. Indeed, by the identity of Lemma 5.2 obtained by duality, we have

$$
\begin{array}{rlr}
\left\|e_{u}\right\|_{\mathcal{T}_{h}}^{2} & =\left(\boldsymbol{q}-\boldsymbol{\Pi}_{V} \boldsymbol{q}, \boldsymbol{\Pi}_{V}^{*} \boldsymbol{\theta}\right)_{\mathcal{T}_{h}}-\left(\boldsymbol{e}_{q}, \boldsymbol{\theta}-\boldsymbol{\Pi}_{V}^{*} \boldsymbol{\theta}\right)_{\mathcal{T}_{h}} \\
& \leq\left\|\boldsymbol{q}-\boldsymbol{\Pi}_{V} \boldsymbol{q}\right\|_{\mathcal{T}_{h}}\left\|\boldsymbol{\Pi}_{V}^{*} \boldsymbol{\theta}\right\|_{\mathcal{T}_{h}}+\left\|\boldsymbol{e}_{q}\right\|_{\mathcal{T}_{h}}\left\|\boldsymbol{\theta}-\boldsymbol{\Pi}_{V}^{*} \boldsymbol{\theta}\right\|_{\mathcal{T}_{h}} & \\
& \leq\left\|\boldsymbol{q}-\boldsymbol{\Pi}_{V} \boldsymbol{q}\right\|_{\mathcal{T}_{h}}\left(\left\|\boldsymbol{\Pi}_{V}^{*} \boldsymbol{\theta}\right\|_{\mathcal{T}_{h}}+\left\|\boldsymbol{\theta}-\boldsymbol{\Pi}_{V}^{*} \boldsymbol{\theta}\right\|_{\mathcal{T}_{h}}\right) & \text { by Theorem 2.1] } \\
& \leq\left\|\boldsymbol{q}-\boldsymbol{\Pi}_{V} \boldsymbol{q}\right\|_{\mathcal{T}_{h}}\left(\|\theta\|_{\mathcal{T}_{h}}+2 C_{a p p} h\left(|\phi|_{H^{2}\left(\mathcal{T}_{h}\right)}+\mid \boldsymbol{\theta} \|_{H^{1}\left(\mathcal{T}_{h}\right)}\right)\right.
\end{array}
$$

by Assumption (B.1). Finally, after a simple application of the elliptic regularity inequality (2.1) with $\eta:=e_{u}$, we get that

$$
\left\|e_{u}\right\|_{\mathcal{T}_{h}} \leq C\left\|\boldsymbol{q}-\boldsymbol{\Pi}_{V} \boldsymbol{q}\right\|_{\mathcal{T}_{h}} .
$$

Thus, even though the Assumption (B.2) does not hold. The convergence in the scalar variable can be optimal.

6.3. Superconvergence when the local space $W(K)$ is small. Next, we show that it is still possible to obtain superconvergence of the projection of the error in the scalar variable when the local space $W(K)$ is small, that is, when it does not satisfy Assumption (B.2). We do this for the $\mathrm{RT}_{0}$ method, which is the only method for which this is known to happen. We begin by noting that, by the identity of Lemma 5.2, we have

$$
\begin{array}{rlr}
\left\|e_{u}\right\|_{\mathcal{T}_{h}}^{2}= & \left(\boldsymbol{q}-\boldsymbol{\Pi}_{V} \boldsymbol{q}, \boldsymbol{\Pi}_{V}^{*} \boldsymbol{\theta}\right)_{\mathcal{T}_{h}}-\left(\boldsymbol{e}_{q}, \boldsymbol{\theta}-\boldsymbol{\Pi}_{V}^{*} \boldsymbol{\theta}\right)_{\mathcal{T}_{h}} \\
= & \left(\boldsymbol{q}-\boldsymbol{\Pi}_{V} \boldsymbol{q}, \boldsymbol{\theta}\right)_{\mathcal{T}_{h}}-\left(\boldsymbol{q}-\boldsymbol{q}_{h}, \boldsymbol{\theta}-\boldsymbol{\Pi}_{V}^{*} \boldsymbol{\theta}\right)_{\mathcal{T}_{h}} \\
= & -\left(\boldsymbol{q}-\boldsymbol{\Pi}_{V} \boldsymbol{q}, \nabla \phi\right)_{\mathcal{T}_{h}}-\left(\boldsymbol{q}-\boldsymbol{q}_{h}, \boldsymbol{\theta}-\boldsymbol{\Pi}_{V}^{*} \boldsymbol{\theta}\right)_{\mathcal{T}_{h}} \\
= & -\left(\boldsymbol{q}-\boldsymbol{\Pi}_{V} \boldsymbol{q}, \nabla(\phi-\bar{\phi})\right)_{\mathcal{T}_{h}}-\left(\boldsymbol{q}-\boldsymbol{q}_{h}, \boldsymbol{\theta}-\boldsymbol{\Pi}_{V}^{*} \boldsymbol{\theta}\right)_{\mathcal{T}_{h}} \\
= & \left(\nabla \cdot\left(\boldsymbol{q}-\boldsymbol{\Pi}_{V} \boldsymbol{q}\right), \phi-\bar{\phi}\right)_{\mathcal{T}_{h}}-\left\langle\left(\boldsymbol{q}-\boldsymbol{\Pi}_{V} \boldsymbol{q}\right) \cdot \boldsymbol{n}, \phi-\bar{\phi}\right\rangle_{\partial \Omega_{h}} \\
& -\left(\boldsymbol{q}-\boldsymbol{q}_{h}, \boldsymbol{\theta}-\boldsymbol{\Pi}_{V}^{*} \boldsymbol{\theta}\right)_{\mathcal{T}_{h}} \\
= & \left(\nabla \cdot\left(\boldsymbol{q}-\boldsymbol{\Pi}_{V} \boldsymbol{q}\right), \phi-\bar{\phi}\right)_{\mathcal{T}_{h}}-\left\langle\left(\boldsymbol{q}-\boldsymbol{\Pi}_{V} \boldsymbol{q}\right) \cdot \boldsymbol{n}, \phi-\bar{\phi}\right\rangle_{\partial \Omega_{h} \backslash \partial \Omega} \\
& -\left(\boldsymbol{q}-\boldsymbol{q}_{h}, \boldsymbol{\theta}-\boldsymbol{\Pi}_{V}^{*} \boldsymbol{\theta}\right)_{\mathcal{T}_{h}}
\end{array}
$$

by the boundary condition of the dual problem (2.2c). For the $\mathrm{RT}_{0}$ method, we can write

$$
\left\|e_{u}\right\|_{\mathcal{T}_{h}}^{2}=(\nabla \cdot \boldsymbol{q}-\overline{\nabla \cdot \boldsymbol{q}}, \phi-\bar{\phi})_{\mathcal{T}_{h}}-\left(\boldsymbol{q}-\boldsymbol{q}_{h}, \boldsymbol{\theta}-\boldsymbol{\Pi}_{V}^{*} \boldsymbol{\theta}\right)_{\mathcal{T}_{h}}
$$


and, proceeding in the previous subsection, we can obtain that

$$
\left\|e_{u}\right\|_{\mathcal{T}_{h}} \leq C h^{2}|\nabla \cdot \boldsymbol{q}|_{H^{1}(\Omega)}+C h\left\|\boldsymbol{q}-\boldsymbol{\Pi}_{V} \boldsymbol{q}\right\|_{\mathcal{T}_{h}} .
$$

Superconvergence of order two is thus achieved for the projection of the error $e_{u}$.

6.4. Other formulas for the numerical trace of the flux. The hybridizable DG method based on the use of the so-called interior penalty (IP) method on each element (see [10]) does not use the formula for the numerical trace of the flux (1.3). Instead it uses the formula

$$
\widehat{\boldsymbol{q}}_{h} \cdot \boldsymbol{n}=-\nabla u_{h} \cdot \boldsymbol{n}+\alpha\left(u_{h}-\widehat{u}_{h}\right) \quad \text { on } \quad \partial \mathcal{T}_{h} .
$$

The application of our approach to this method remains open. However, let us point out that since when $\alpha=\tau$, this method is well defined provided $\tau$ is of order $1 / h$; see [10. As a consequence, it seems very unlikely that optimal convergence will be attained for the approximate flux.

6.5. Conclusion. The projection-approach we have presented here provides a simple, unified a priori error analysis of a large class of finite elements methods including mixed and HDG methods. It provides sufficient conditions on the different local spaces and by the local stabilization operator $\alpha$ that guarantee the superconvergence of the postprocessing $u_{h}^{*}$. In other words, it gives us guidelines for the devising of new superconvergent methods for elliptic problems.

We have also proposed a template to construct such methods and have shown that all previously known mixed methods and the $\mathbf{H D G}_{k}$ methods for simplexes with the local stabilization operator used in [3, 7, 10, fit in it. We have also shown how to use it to uncover several superconvergent $\mathbf{H D G}_{[k]}$ methods for squares, cubes and prisms; they are the only DG methods using those elements known to be superconvergent). We have also used the template to uncover what seems to be the smallest superconvergent mixed method, on squares and cubes, containing the tensor-product space $\boldsymbol{Q}^{k}(K) \times Q^{k}(K)$.

The extension of this approach to the Stokes system of incompressible fluid flow constitutes the subject of ongoing work.

\section{REFERENCES}

[1] D. N. Arnold and F. Brezzi, Mixed and nonconforming finite element methods: implementation, postprocessing and error estimates, RAIRO Modél. Math. Anal. Numér. 19 (1985), 7-32. MR813687 (87g:65126)

[2] D. N. Arnold, F. Brezzi, B. Cockburn, and L. D. Marini, Unified analysis of discontinuous Galerkin methods for elliptic problems, SIAM J. Numer. Anal. 39 (2002), 1749-1779. MR1885715 (2002k:65183)

[3] F. Bassi, S. Rebay, G. Mariotti, S. Pedinotti, and M. Savini, A high-order accurate discontinuous finite element method for inviscid and viscous turbomachinery flows, 2nd European Conference on Turbomachinery Fluid Dynamics and Thermodynamics (Antwerpen, Belgium) (R. Decuypere and G. Dibelius, eds.), Technologisch Instituut, March 5-7 1997, pp. 99-108.

[4] F. Brezzi, J. Douglas, Jr., M. Fortin, and L. D. Marini, Efficient rectangular mixed finite element methods in two and three space variables, RAIRO Modél. Math. Anal. Numér. 21 (1987), 581-604. MR921828 (88j:65249)

[5] F. Brezzi, J. Douglas, Jr., and L. D. Marini, Two families of mixed finite elements for second order elliptic problems, Numer. Math. 47 (1985), 217-235. MR799685(87g:65133)

[6] F. Brezzi and M. Fortin, Mixed and hybrid finite element methods, Springer-Verlag, 1991. MR1115205 (92d:65187) 
[7] F. Brezzi, G. Manzini, L. D. Marini, P. Pietra, and A. Russo, Discontinuous finite elements for diffusion problems, in Atti Convegno in onore di F. Brioschi (Milan 1997), Istituto Lombardo, Accademia di Scienze e Lettere, 1999, pp. 197-217.

[8] C.D. Cantwell, S.J. Sherwin, R.M. Kirby, and P.H.J. Kelly, From $h$ to $p$ efficiency: Strategy selection for operator evaluation on hexahedral and tetrahedral elements, Computers and Fluids 43 (2011), no. 1, pp. 23-28.

[9] B. Cockburn, B. Dong, and J. Guzmán, A superconvergent LDG-hybridizable Galerkin method for second-order elliptic problems, Math. Comp. 77 (2008), 1887-1916. MR 2429868 (2009d:65166)

[10] B. Cockburn, J. Gopalakrishnan, and R. Lazarov, Unified hybridization of discontinuous Galerkin, mixed and continuous Galerkin methods for second order elliptic problems, SIAM J. Numer. Anal. 47 (2009), 1319-1365. MR2485455 (2010b:65251)

[11] B. Cockburn, J. Gopalakrishnan, and F.-J. Sayas, A projection-based error analysis of HDG methods, Math. Comp. 79 (2010), 1351-1367. MR2629996 (2011d:65354)

[12] J. Douglas, Jr. and J.E. Roberts, Global estimates for mixed methods for second order elliptic equations, Math. Comp. 44 (1985), 39-52. MR771029(86b:65122)

[13] L. Gastaldi and R.H. Nochetto, Sharp maximum norm error estimates for general mixed finite element approximations to second order elliptic equations, RAIRO Modél. Math. Anal. Numér. 23 (1989), 103-128. MR.1015921 (91b:65125)

[14] Z. Chen and J. Douglas, Jr. Prismatic mixed finite elements for second order elliptic problems, Calcolo 26 (1989), no. 2-4, 135-148 (1990) MR1083050(92e:65148)

[15] J.-C. Nédélec, Mixed finite elements in $\mathbf{R}^{3}$, Numer. Math. 35 (1980), 315-341. MR592160 (81k:65125)

[16] P. A. Raviart and J. M. Thomas, A mixed finite element method for second order elliptic problems, Mathematical Aspects of Finite Element Method, Lecture Notes in Math. 606 (I. Galligani and E. Magenes, eds.), Springer-Verlag, New York, 1977, pp. 292-315. MR0483555 $(58: 3547)$

[17] R. Stenberg, A family of mixed finite elements for the elasticity problem, Numer. Math. 53 (1988), 513-538. MR954768 (89h:65192)

[18] R. Stenberg, Postprocessing schemes for some mixed finite elements, RAIRO Modél. Math. Anal. Numér. 25 (1991), 151-167. MR1086845 (92a:65303)

[19] P.E.J. Vos, S.J. Sherwin, and R.M. Kirby, From $h$ to $p$ efficiency: Implementing finite and spectral/hp element methods to achieve optimal performance for low- and high-order discretizations, J. Comput. Phys. 229 (2010), 5161-5181. MR2643647

[20] T. Warburton, L. F. Pavarino, and J. S. Hesthaven, A pseudo-spectral scheme for the incompressible Navier-Stokes equations using unstructured nodal elements, J. Comput. Phys. 164 (2000), 1-21. MR 1786240 (2001f:76062)

School of Mathematics, University of Minnesota, Minneapolis, Minnesota 55455

E-mail address: cockburn@math.umn.edu

Institute for Mathematics and its Applications, University of Minnesota, MinneapoLis, Minnesota 55455

E-mail address: qiuxa001@ima.umn.edu

School of Mathematics, University of Minnesota, Minneapolis, Minnesota 55455

E-mail address: shixx075@math.umn.edu 